\title{
Charts, signatures, and stabilizations of Lefschetz fibrations
}

\author{
HISAAKI ENDO \\ ISAO HASEGAWA \\ SEIICHI KAMADA \\ KOKORO TANAKA
}

\begin{abstract}
We employ a certain labeled finite graph, called a chart, in a closed oriented surface to describe the monodromy of a(n achiral) Lefschetz fibration over the surface. Applying charts and their moves with respect to Wajnryb's presentation of mapping class groups, we first generalize a signature formula for Lefschetz fibrations over the 2-sphere obtained by Endo and Nagami to that for Lefschetz fibrations over arbitrary closed oriented surface. We then prove two theorems on stabilization of Lefschetz fibrations under fiber summing with copies of a typical Lefschetz fibration as generalizations of a theorem of Auroux.
\end{abstract}

57M15; 57N13

\section{Introduction}

Matsumoto [31] proved that every Lefschetz fibration of genus one over a closed oriented surface is isomorphic to a fiber sum of copies of a holomorphic elliptic fibration on $\mathbb{C P}^{2} \# 9 \overline{\mathbb{C P}}^{2}$ and a trivial torus bundle over the surface if it has at least one critical point. This result played a crucial role in completing the classification of diffeomorphism types of elliptic surfaces (see Gompf and Stipsicz [13, Section 8.3]). Although such a classification has not been established for Lefschetz fibrations of higher genus, Auroux [1] proved a stabilization theorem for Lefschetz fibrations of genus two, which states that every Lefschetz fibration of genus two over the 2-sphere becomes isomorphic to a fiber sum of copies of three typical fibrations after fiber summing with a holomorphic fibration on $\mathbb{C P}^{2} \# 13 \overline{\mathbb{C P}}^{2}$. Auroux [2] gave a generalization of this theorem for Lefschetz fibrations of higher genus, which states that two Lefschetz fibrations of the same genus over the 2-sphere which have the same signature, the same numbers of singular fibers of each type, and admit sections of the same selfintersection number become isomorphic after fiber summing the same number of copies of a "universal" Lefschetz fibration. 
Kamada [17; 18] introduced charts, which are labeled finite graphs in a disk, to describe monodromies of surface braids (see also a textbook of Kamada [19]). Kamada, Matsumoto, Matumoto, and Waki [22] considered a variant of charts for Lefschetz fibrations of genus one to give a remarkably simple proof of the above result of Matsumoto. Furthermore, Kamada [21] and Endo and Kamada [6; 5] made use of generalized charts to give a simple proof of the above theorem of Auroux for Lefschetz fibrations of genus two, and to investigate a stabilization theorem and an invariant of hyperelliptic Lefschetz fibrations of arbitrary genus. See also Baykur and Kamada [4], and Hayano [16] for applications of charts to broken Lefschetz fibrations.

In this paper we introduce a chart description for Lefschetz fibrations of genus greater than two over closed oriented surfaces of arbitrary genus to show a signature formula and two theorems on stabilization for such fibrations. In Section 2 we introduce charts and chart moves with respect to Wajnryb's presentation of mapping class groups to examine monodromies of Lefschetz fibrations. After a short survey of Meyer's signature cocycle, we generalize a signature formula of Endo and Nagami [8] for Lefschetz fibrations over the 2-sphere to that for Lefschetz fibrations over a closed oriented surface of arbitrary genus in Section 3. Section 4 is devoted to proofs of two theorems on stabilization of Lefschetz fibrations under fiber summing with copies of a "universal" Lefschetz fibration. In particular the first of our stabilization theorems is a generalization of the theorem of Auroux [2]. We make several comments on variations of chart description and propose some possible directions for future research in Section 5.

\section{Chart description for Lefschetz fibrations}

In this section we review a definition and properties of Lefschetz fibrations and introduce a chart description for Lefschetz fibrations of genus greater than two.

\subsection{Lefschetz fibrations and their monodromies}

In this subsection we review a precise definition and basic properties of Lefschetz fibrations. More details can be found in Matsumoto [32] and Gompf and Stipsicz [13]. Let $\Sigma_{g}$ be a connected closed oriented surface of genus $g$.

Definition 2.1 Let $M$ and $B$ be connected closed oriented smooth 4-manifold and 2-manifold, respectively. A smooth map $f: M \rightarrow B$ is called a Lefschetz fibration of genus $g$ if it satisfies the following conditions:

(i) The set $\Delta \subset B$ of critical values of $f$ is finite and $f$ is a smooth fiber bundle over $B-\Delta$ with fiber $\Sigma_{g}$. 
(ii) For each $b \in \Delta$, there exists a unique critical point $p$ in the singular fiber $F_{b}:=f^{-1}(b)$ such that $f$ is locally written as $f\left(z_{1}, z_{2}\right)=z_{1} z_{2}$ or $\bar{z}_{1} z_{2}$ with respect to some local complex coordinates around $p$ and $b$ which are compatible with the orientations of $M$ and $B$.

(iii) No fiber contains a \pm 1 -sphere.

We call $M$ the total space, $B$ the base space, and $f$ the projection. We call $p$ a critical point of positive type (resp. of negative type) and $F_{b}$ a singular fiber of positive type (resp. of negative type) if $f$ is locally written as $f\left(z_{1}, z_{2}\right)=z_{1} z_{2}$ (resp. $\left.f\left(z_{1}, z_{2}\right)=\bar{z}_{1} z_{2}\right)$ in (ii). For a regular value $b \in B$ of $f, f^{-1}(b)$ is often called a general fiber.

Remark 2.2 A Lefschetz fibration in this paper is called an achiral Lefschetz fibration in many other papers.

Let $f: M \rightarrow B$ and $f^{\prime}: M^{\prime} \rightarrow B$ be Lefschetz fibrations of genus $g$ over the same base space $B$. We say that $f$ is isomorphic to $f^{\prime}$ if there exist orientation-preserving diffeomorphisms $H: M \rightarrow M^{\prime}$ and $h: B \rightarrow B$ which satisfy $f^{\prime} \circ H=h \circ f$. If we can choose such an $h$ isotopic to the identity relative to a given base point $b_{0} \in B$, we say that $f$ is strictly isomorphic to $f^{\prime}$.

Let $\mathcal{M}_{g}$ be the mapping class group of $\Sigma_{g}$, namely, the group of all isotopy classes of orientation-preserving diffeomorphisms of $\Sigma_{g}$. We assume that $\mathcal{M}_{g}$ acts on the right: the symbol $\varphi \psi$ means that we apply $\varphi$ first and then $\psi$ for $\varphi, \psi \in \mathcal{M}_{g}$. We denote the mapping class group of $\Sigma_{g}$ acting on the left by $\mathcal{M}_{g}^{*}$. Hence the identity map $\mathcal{M}_{g} \rightarrow \mathcal{M}_{g}^{*}$ is an anti-isomorphism.

Let $f: M \rightarrow B$ be a Lefschetz fibration of genus $g$ as in Definition 2.1. Take a base point $b_{0} \in B$ and an orientation-preserving diffeomorphism $\Phi: \Sigma_{g} \rightarrow F_{0}:=f^{-1}\left(b_{0}\right)$. Since $f$ restricted over $B-\Delta$ is a smooth fiber bundle with fiber $\Sigma_{g}$, we can define a homomorphism

$$
\rho: \pi_{1}\left(B-\Delta, b_{0}\right) \rightarrow \mathcal{M}_{g}
$$

called the monodromy representation of $f$ with respect to $\Phi$. Let $\gamma$ be the loop based at $b_{0}$ consisting of the boundary circle of a small disk neighborhood of $b \in \Delta$ oriented counterclockwise and a simple path connecting a point on the circle to $b_{0}$ in $B-\Delta$. It is known that $\rho([\gamma])$ is a Dehn twist along some essential simple closed curve $c$ on $\Sigma_{g}$, which is called the vanishing cycle of the critical point $p$ on $f^{-1}(b)$. If $p$ is of positive type (resp. of negative type), then the Dehn twist is right-handed (resp. left-handed). 
A singular fiber is said to be of type I if the vanishing cycle is non-separating and of type $\mathrm{II}_{h}$ for $h=1, \ldots,[g / 2]$ if the vanishing cycle is separating and it bounds a genus- $h$ subsurface of $\Sigma_{g}$. A singular fiber is said to be of type $\mathrm{I}^{+}$(resp. type $\mathrm{I}^{-}$and type $\mathrm{II}_{h}^{+}$, type $\mathrm{II}_{h}^{-}$) if it is of type I and of positive type (resp. of type I and of negative type, of type $\mathrm{II}_{h}$ and of positive type, of type $\mathrm{II}_{h}$ and of negative type). We denote by $n_{0}^{+}(f), n_{0}^{-}(f), n_{h}^{+}(f)$, and $n_{h}^{-}(f)$, the numbers of singular fibers of $f$ of type $\mathrm{I}^{+}, \mathrm{I}^{-}, \mathrm{II}_{h}^{+}$, and $\mathrm{II}_{h}^{-}$, respectively. A Lefschetz fibration is called irreducible if every singular fiber is of type I. A Lefschetz fibration is called chiral if every singular fiber is of positive type.

Suppose that the cardinality of $\Delta$ is equal to $n$. A system $\mathcal{A}=\left(A_{1}, \ldots, A_{n}\right)$ of arcs on $B$ is called a Hurwitz arc system for $\Delta$ with base point $b_{0}$ if each $A_{i}$ is an embedded arc connecting $b_{0}$ with a point of $\Delta$ in $B$ such that $A_{i} \cap A_{j}=\left\{b_{0}\right\}$ for $i \neq j$, and they appear in this order around $b_{0}$ (see Kamada [19]). When $B$ is a $2-$ sphere, the system $\mathcal{A}$ determines a system of generators of $\pi_{1}\left(B-\Delta, b_{0}\right)$, say $\left(a_{1}, \ldots, a_{n}\right)$. We call $\left(\rho\left(a_{1}\right), \ldots, \rho\left(a_{n}\right)\right)$ a Hurwitz system of $f$.

\subsection{Chart description and Wajnryb's presentation}

In this subsection we introduce a chart description for Lefschetz fibrations of genus greater than two by employing Wajnryb's finite presentation [40] of mapping class groups. General theories of charts for presentations of groups were developed independently by Kamada [20] and Hasegawa [15]. We use the terminology of chart description in Kamada [20].

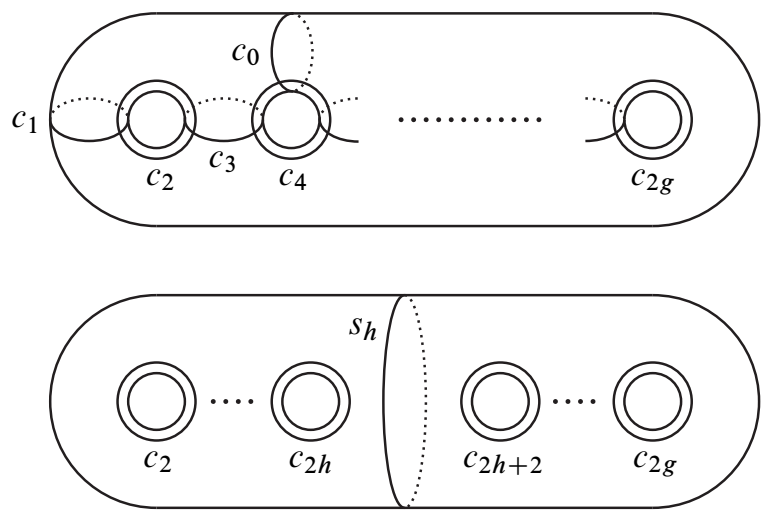

Figure 1: Simple closed curves on $\Sigma_{g}$

We first review a finite presentation of the mapping class group of a closed oriented surface due to Wajnryb. For $i=0,1, \ldots, 2 g$, let $\zeta_{i}$ be a right-handed Dehn twist along the simple closed curve $c_{i}$ on $\Sigma_{g}$ depicted in Figure 1. 
Theorem 2.3 (Wajnryb [40; 41]) Suppose that $g$ is greater than two. The mapping class group $\mathcal{M}_{g}$ is generated by the elements $\zeta_{0}, \zeta_{1}, \zeta_{2}, \ldots, \zeta_{2 g}$ and has the following defining relations:

- (Far commutation)

$$
\begin{array}{ll}
\zeta_{i} \zeta_{j}=\zeta_{j} \zeta_{i} & (1 \leq i<j-1 \leq 2 g-1) \\
\zeta_{0} \zeta_{j}=\zeta_{j} \zeta_{0} & (j=1,2,3,5, \ldots, 2 g)
\end{array}
$$

- (Braid relation)

$$
\begin{aligned}
\zeta_{i} \zeta_{i+1} \zeta_{i} & =\zeta_{i+1} \zeta_{i} \zeta_{i+1} \quad(i=1, \ldots, 2 g-1) \\
\zeta_{0} \zeta_{4} \zeta_{0} & =\zeta_{4} \zeta_{0} \zeta_{4}
\end{aligned}
$$

- (3-chain relation)

$$
\left(\zeta_{3} \zeta_{2} \zeta_{1}\right)^{4}=\zeta_{0} \zeta_{4}^{-1} \zeta_{3}^{-1} \zeta_{2}^{-1} \zeta_{1}^{-2} \zeta_{2}^{-1} \zeta_{3}^{-1} \zeta_{4}^{-1} \zeta_{0} \zeta_{4} \zeta_{3} \zeta_{2} \zeta_{1}^{2} \zeta_{2} \zeta_{3} \zeta_{4}
$$

- (Lantern relation)

$$
\delta_{3} \zeta_{1} \zeta_{3} \zeta_{5}=\zeta_{0} \tau_{2} \zeta_{0} \tau_{2}^{-1} \tau_{1} \tau_{2} \zeta_{0} \tau_{2}^{-1} \tau_{1}^{-1}
$$

where

$$
\begin{gathered}
\tau_{1}:=\zeta_{2} \zeta_{3} \zeta_{1} \zeta_{2}, \quad \tau_{2}:=\zeta_{4} \zeta_{5} \zeta_{3} \zeta_{4}, \quad \mu:=\zeta_{5} \zeta_{6} \tau_{2} \zeta_{0} \tau_{2}^{-1} \zeta_{6}^{-1} \zeta_{5}^{-1}, \\
v:=\zeta_{1} \zeta_{2} \zeta_{3} \zeta_{4} \zeta_{0} \zeta_{4}^{-1} \zeta_{3}^{-1} \zeta_{2}^{-1} \zeta_{1}^{-1}, \quad \delta_{3}:=\zeta_{6}^{-1} \zeta_{5}^{-1} \zeta_{4}^{-1} \zeta_{3}^{-1} \zeta_{2}^{-1} \mu^{-1} v \mu \zeta_{2} \zeta_{3} \zeta_{4} \zeta_{5} \zeta_{6} .
\end{gathered}
$$

- (Hyperelliptic relation)

$$
\zeta_{2 g} \cdots \zeta_{3} \zeta_{2} \zeta_{1}^{2} \zeta_{2} \zeta_{3} \cdots \zeta_{2 g} \delta_{g}=\delta_{g} \zeta_{2 g} \cdots \zeta_{3} \zeta_{2} \zeta_{1}^{2} \zeta_{2} \zeta_{3} \cdots \zeta_{2 g}
$$

where

$$
\begin{aligned}
& \tau_{1}:=\zeta_{2} \zeta_{3} \zeta_{1} \zeta_{2} \text {, } \\
& \tau_{i}:=\zeta_{2 i} \zeta_{2 i-1} \zeta_{2 i+1} \zeta_{2 i}, \\
& v_{1}:=\zeta_{4}^{-1} \zeta_{3}^{-1} \zeta_{2}^{-1} \zeta_{1}^{-2} \zeta_{2}^{-1} \zeta_{3}^{-1} \zeta_{4}^{-1} \zeta_{0} \zeta_{4} \zeta_{3} \zeta_{2} \zeta_{1}^{2} \zeta_{2} \zeta_{3} \zeta_{4}, \quad v_{i}:=\tau_{i-1} \tau_{i} v_{i-1} \tau_{i}^{-1} \tau_{i-1}^{-1} \text {, } \\
& \mu_{1}:=\zeta_{2} \zeta_{3} \zeta_{4} v_{1} \zeta_{1}^{-1} \zeta_{2}^{-1} \zeta_{3}^{-1} \zeta_{4}^{-1} \\
& \mu_{i}:=\zeta_{2 i} \zeta_{2 i+1} \zeta_{2 i+2} v_{i} \zeta_{2 i-1}^{-1} \zeta_{2 i}^{-1} \zeta_{2 i+1}^{-1} \zeta_{2 i+2}^{-1} \text {, } \\
& \delta_{g}:=\mu_{g-1}^{-1} \cdots \mu_{2}^{-1} \mu_{1}^{-1} \zeta_{1} \mu_{1} \mu_{2} \cdots \mu_{g-1} \text {, }
\end{aligned}
$$

for $i=2, \ldots, g-1$. 
We make use of the presentation above to introduce a notion of chart which gives a graphic description of monodromy representations of Lefschetz fibrations. We set

$$
\begin{aligned}
& \mathcal{X}:=\left\{\zeta_{0}, \zeta_{1}, \ldots, \zeta_{2 g}\right\}, \\
& \mathcal{R}:=\left\{r_{F}(i, j) \mid 1 \leq i<j-1 \leq 2 g-1\right\} \cup\left\{r_{F}(0, j) \mid j=1,2,3,5, \ldots, 2 g\right\} \\
& \cup\left\{r_{B}(i) \mid i=0,1, \ldots, 2 g-1\right\} \cup\left\{r_{C}, r_{L}, r_{H}\right\}, \\
& \mathcal{S}:=\left\{\ell_{0}(i)^{ \pm 1} \mid i=0,1, \ldots, 2 g\right\} \cup\left\{\ell_{h}^{ \pm 1} \mid h=1, \ldots,[g / 2]\right\},
\end{aligned}
$$

for $g \geq 3$, where

$$
\begin{aligned}
r_{F}(i, j) & :=\zeta_{i} \zeta_{j} \zeta_{i}^{-1} \zeta_{j}^{-1}, \\
r_{B}(0) & :=\zeta_{0} \zeta_{4} \zeta_{0} \zeta_{4}^{-1} \zeta_{0}^{-1} \zeta_{4}^{-1}, \\
r_{B}(i) & :=\zeta_{i} \zeta_{i+1} \zeta_{i} \zeta_{i+1}^{-1} \zeta_{i}^{-1} \zeta_{i+1}^{-1} \quad(i=1, \ldots, 2 g-1), \\
r_{C} & :=\left(\zeta_{3} \zeta_{2} \zeta_{1}\right)^{4} \zeta_{4}^{-1} \zeta_{3}^{-1} \zeta_{2}^{-1} \zeta_{1}^{-2} \zeta_{2}^{-1} \zeta_{3}^{-1} \zeta_{4}^{-1} \zeta_{0}^{-1} \zeta_{4} \zeta_{3} \zeta_{2} \zeta_{1}^{2} \zeta_{2} \zeta_{3} \zeta_{4} \zeta_{0}^{-1}, \\
r_{L} & :=\delta_{3} \zeta_{1} \zeta_{3} \zeta_{5} \tau_{1} \tau_{2} \zeta_{0}^{-1} \tau_{2}^{-1} \tau_{1}^{-1} \tau_{2} \zeta_{0}^{-1} \tau_{2}^{-1} \zeta_{0}^{-1}, \\
r_{H} & :=\zeta_{2 g} \cdots \zeta_{3} \zeta_{2} \zeta_{1}^{2} \zeta_{2} \zeta_{3} \cdots \zeta_{2 g} \delta_{g} \zeta_{2 g}^{-1} \cdots \zeta_{3}^{-1} \zeta_{2}^{-1} \zeta_{1}^{-2} \zeta_{2}^{-1} \zeta_{3}^{-1} \ldots \zeta_{2 g}^{-1} \delta_{g}^{-1}, \\
\ell_{0}(i) & :=\zeta_{i} \quad(i=0,1, \ldots, 2 g), \\
\ell_{h} & :=\left(\zeta_{1} \zeta_{2} \cdots \zeta_{2 h}\right)^{4 h+2} \quad(h=1, \ldots,[g / 2]),
\end{aligned}
$$

and $\delta_{3}, \tau_{1}, \tau_{2}, \delta_{g}$ are defined as in Theorem 2.3.

Let $B$ be a connected closed oriented surface and $\Gamma$ a finite graph in $B$ such that each edge of $\Gamma$ is oriented and labeled with an element of $\mathcal{X}$. We denote the label $\zeta_{i}$ by $i$ for short. Choose a simple path $\gamma$ which intersects with edges of $\Gamma$ transversely and does not intersect with vertices of $\Gamma$. For such a path $\gamma$, we obtain a word $w_{\Gamma}(\gamma)$ in $\mathcal{X} \cup \mathcal{X}^{-1}$ by reading off the labels of intersecting edges along $\gamma$ with exponents as in Figure 2(a). We call the word $w_{\Gamma}(\gamma)$ the intersection word of $\gamma$ with respect to $\Gamma$. Conversely, we can specify the number, orientations, and labels of consecutive edges in $\Gamma$ by indicating a (dashed) arrow intersecting the edges transversely together with the intersection word of the arrow with respect to $\Gamma$ (see Figure 2(b) and (c)).

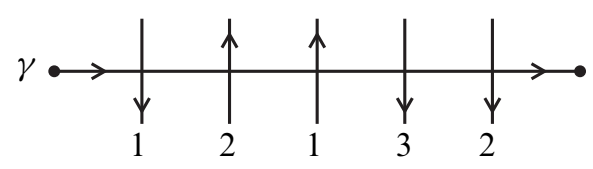

(a)

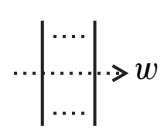

(b)

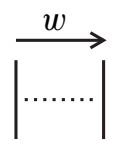

(c)

Figure 2: Intersection word $w_{\Gamma}(\gamma)=w=\zeta_{1} \zeta_{2}^{-1} \zeta_{1}^{-1} \zeta_{3} \zeta_{2}$ 
For a vertex $v$ of $\Gamma$, a small simple closed curve surrounding $v$ in the counterclockwise direction is called a meridian loop of $v$ and denoted by $m_{v}$. The vertex $v$ is said to be marked if one of the regions around $v$ is specified by an asterisk. If $v$ is marked, the intersection word $w_{\Gamma}\left(m_{v}\right)$ of $m_{v}$ with respect to $\Gamma$ is well-defined. If not, it is determined up to cyclic permutation. See Kamada [20] for details.

Definition 2.4 A chart in $B$ is a finite graph $\Gamma$ in $B$ (possibly being empty or having hoops that are closed edges without vertices) whose edges are labeled with an element of $\mathcal{X}$, and oriented so that the following conditions are satisfied (see Figures 3-5):

(1) The vertices of $\Gamma$ are classified into two families: white vertices and black vertices.

(2) If $v$ is a white vertex (resp. a black vertex), the word $w_{\Gamma}\left(m_{v}\right)$ is a cyclic permutation of an element of $\mathcal{R} \cup \mathcal{R}^{-1}$ (resp. of $\mathcal{S}$ ).

A white vertex $v$ is said to be of type $r$ (resp. of type $r^{-1}$ ) if $w_{\Gamma}\left(m_{v}\right)^{-1}$ is a cyclic permutation of $r \in \mathcal{R}$ (resp. of $r^{-1} \in \mathcal{R}^{-1}$ ). A black vertex $v$ is said to be of type $s$ if $w_{\Gamma}\left(m_{v}\right)$ is a cyclic permutation of $s \in \mathcal{S}$. A chart $\Gamma$ is said to be marked if each white vertex (resp. black vertex) $v$ is marked and $w_{\Gamma}\left(m_{v}\right)$ is exactly an element of $\mathcal{R} \cup \mathcal{R}^{-1}$ (resp. of $\mathcal{S}$ ). If a base point $b_{0}$ of $B$ is specified, we always assume that a chart $\Gamma$ is disjoint from $b_{0}$. A chart consisting of two black vertices and one edge connecting them is called a free edge.
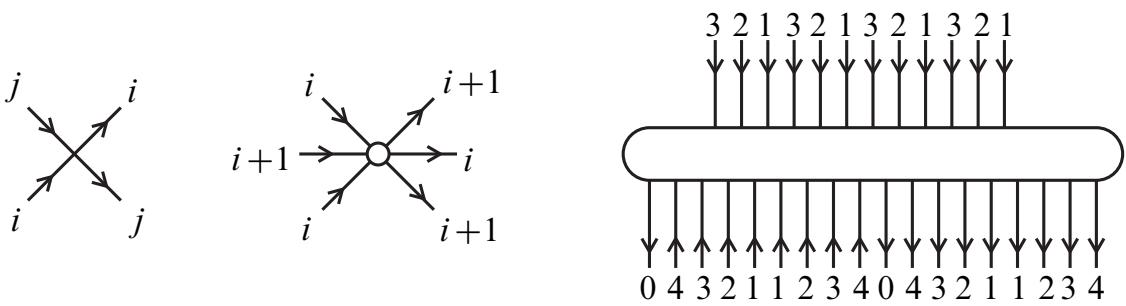

Figure 3: Vertices of type $r_{F}(i, j), r_{B}(i)(i \neq 0), r_{C}$
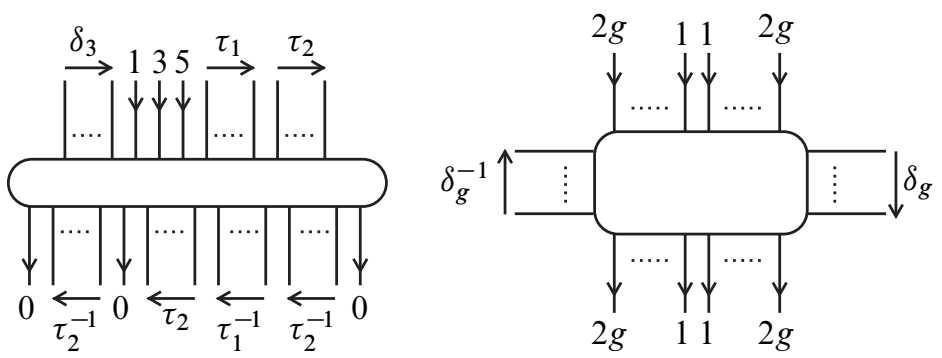

Figure 4: Vertices of type $r_{L}$ and $r_{H}$ 

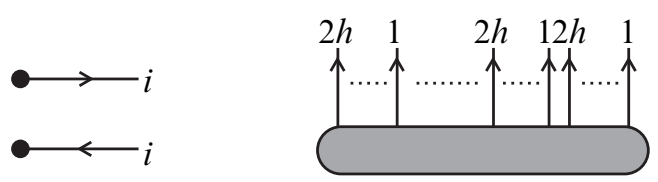

Figure 5: Vertices of type $\ell_{0}(i)^{ \pm 1}$ and $\ell_{h}$

Remark 2.5 It is worth noting that the intersection word of a "clockwise" meridian of a white vertex of type $r$ is equal to $r$, while that of a "counterclockwise" meridian of a black vertex of type $s$ is equal to $s$ in this paper. This notation is different from those of Kamada [20] and Hasegawa [15], who always consider "counterclockwise" meridians for both white and black vertices.

We next introduce several moves for charts. Let $\Gamma$ and $\Gamma^{\prime}$ be two charts on $B$ and $b_{0}$ a base point of $B$.

Let $D$ be a disk embedded in $B-\left\{b_{0}\right\}$. Suppose that the boundary $\partial D$ of $D$ intersects $\Gamma$ and $\Gamma^{\prime}$ transversely.

Definition 2.6 We say that $\Gamma^{\prime}$ is obtained from $\Gamma$ by a chart move of type $W$ if $\Gamma \cap(B-$ Int $D)=\Gamma^{\prime} \cap(B-$ Int $D)$ and both $\Gamma \cap D$ and $\Gamma^{\prime} \cap D$ have no black vertices. We call chart moves of type $\mathrm{W}$ shown in Figure 6(a)-(c) a channel change, a birth/death of a hoop, and a birth/death of a pair of white vertices, respectively.

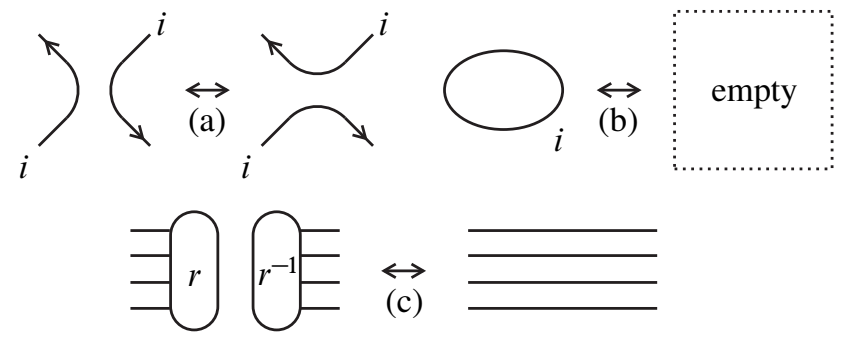

Figure 6: Chart moves of type W

Let $s$ and $s^{\prime}$ be elements of $\mathcal{S}$. Suppose that there exists a word $w$ in $\mathcal{X} \cup \mathcal{X}^{-1}$ such that two words $s^{\prime}$ and $w s w^{-1}$ determine the same element of $\mathcal{M}_{g}$.

Definition 2.7 If a chart $\Gamma$ contains a black vertex of type $s$, then we can change a part of $\Gamma$ near the vertex by using a local replacement depicted in Figure 7 to obtain another chart $\Gamma^{\prime}$. We say that $\Gamma^{\prime}$ is obtained from $\Gamma$ by a chart move of transition. Note that the blank labeled with $\mathrm{T}$ can be filled only with edges and white vertices. 

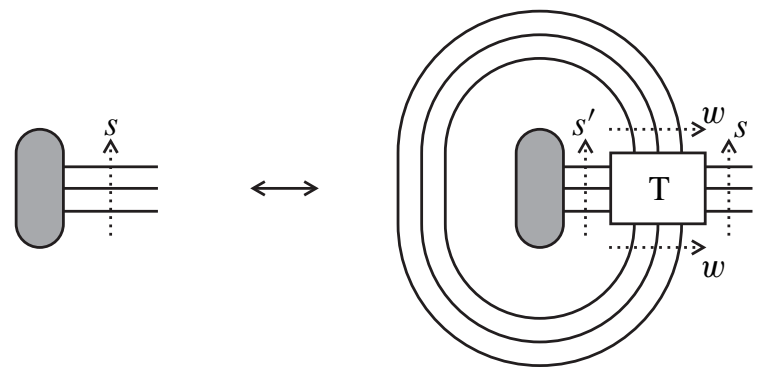

Figure 7: Chart move of transition

Definition 2.8 We say that $\Gamma^{\prime}$ is obtained from $\Gamma$ by a chart move of conjugacy type if $\Gamma^{\prime}$ is obtained from $\Gamma$ by a local replacement depicted in Figure 8.

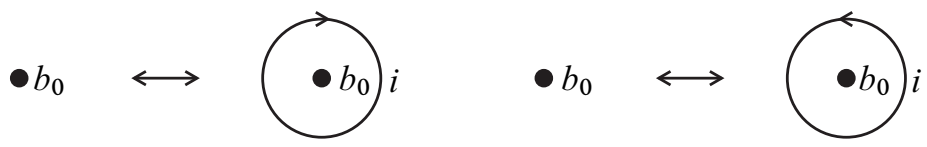

Figure 8: Chart moves of conjugacy type

Let $\Gamma$ be a chart in $B$ with base point $b_{0}$ and $\Delta_{\Gamma}$ the set of black vertices of $\Gamma$. For a loop $\gamma$ in $B-\Delta_{\Gamma}$ based at $b_{0}$, the element of $\mathcal{M}_{g}$ determined by the intersection word $w_{\Gamma}(\gamma)$ of $\gamma$ with respect to $\Gamma$ does not depend on a choice of representative of the homotopy class of $\gamma$. Thus we obtain a homomorphism $\rho_{\Gamma}: \pi_{1}\left(B-\Delta_{\Gamma}, b_{0}\right) \rightarrow \mathcal{M}_{g}$, which is called the homomorphism determined by $\Gamma$.

We now state a classification of Lefschetz fibrations in terms of charts and chart moves. Let $B$ be a connected closed oriented surface.

Proposition 2.9 Suppose that $g$ is greater than two.

(1) Let $f$ be a Lefschetz fibration of genus $g$ over $B$ and $\rho$ a monodromy representation of $f$. Then there exists a chart $\Gamma$ in $B$ such that the homomorphism $\rho_{\Gamma}$ determined by $\Gamma$ is equal to $\rho$.

(2) For every chart $\Gamma$ in $B$, there exists a Lefschetz fibration $f$ of genus $g$ over $B$ such that a monodromy representation of $f$ is equal to the homomorphism $\rho_{\Gamma}$ determined by $\Gamma$.

We call such $\Gamma$ as in Proposition 2.9(1) a chart corresponding to $f$, and such $f$ as in Proposition 2.9(2) a Lefschetz fibration described by $\Gamma$.

Instead of giving a proof of Proposition 2.9, we show an example of a chart and describe the correspondence of the chart to a Hurwitz system of a Lefschetz fibration. 
Example 2.10 Let $B$ be a 2-sphere. We consider a chart $\Gamma$ in $B$ with base point $b_{0}$ and a system $\left(\gamma_{1}, \gamma_{2}, \gamma_{3}, \gamma_{4}\right)$ of loops based at $b_{0}$, which is determined by a Hurwitz arc system $\mathcal{A}$ for the set $\Delta_{\Gamma}$ of black vertices of $\Gamma$, as in Figure 9. The intersection words of the loops with respect to $\Gamma$ are

$$
\begin{array}{ll}
w_{\Gamma}\left(\gamma_{1}\right)=\zeta_{1}^{-1} \zeta_{2}^{-1} \zeta_{1} \zeta_{2} \zeta_{1}, & w_{\Gamma}\left(\gamma_{2}\right)=\zeta_{1}^{-1} \zeta_{3} \zeta_{1}, \\
w_{\Gamma}\left(\gamma_{3}\right)=\zeta_{2}^{-1} \zeta_{3}^{-1} \zeta_{2}^{-1} \zeta_{3} \zeta_{2}, & w_{\Gamma}\left(\gamma_{4}\right)=\zeta_{2}^{-1},
\end{array}
$$

each of which represents the image $\rho_{\Gamma}\left(a_{i}\right)$ of the homotopy class $a_{i}$ of $\gamma_{i}$ under the homomorphism $\rho_{\Gamma}: \pi_{1}\left(B-\Delta_{\Gamma}, b_{0}\right) \rightarrow \mathcal{M}_{g}$. Since the group $\pi_{1}\left(B-\Delta_{\Gamma}, b_{0}\right)$ has a presentation $\left\langle a_{1}, a_{2}, a_{3}, a_{4} \mid a_{1} a_{2} a_{3} a_{4}=1\right\rangle, \rho_{\Gamma}$ is determined by the system $\left(\rho_{\Gamma}\left(a_{1}\right), \rho_{\Gamma}\left(a_{2}\right), \rho_{\Gamma}\left(a_{3}\right), \rho_{\Gamma}\left(a_{4}\right)\right)$, which is a Hurwitz system of a certain Lefschetz fibration of genus $g$ over $B$ because each $\rho_{\Gamma}\left(a_{i}\right)$ is a Dehn twist. Note that the product $w_{\Gamma}\left(\gamma_{1}\right) w_{\Gamma}\left(\gamma_{2}\right) w_{\Gamma}\left(\gamma_{3}\right) w_{\Gamma}\left(\gamma_{4}\right)$ of the intersection words represents the identity of $\mathcal{M}_{g}$.

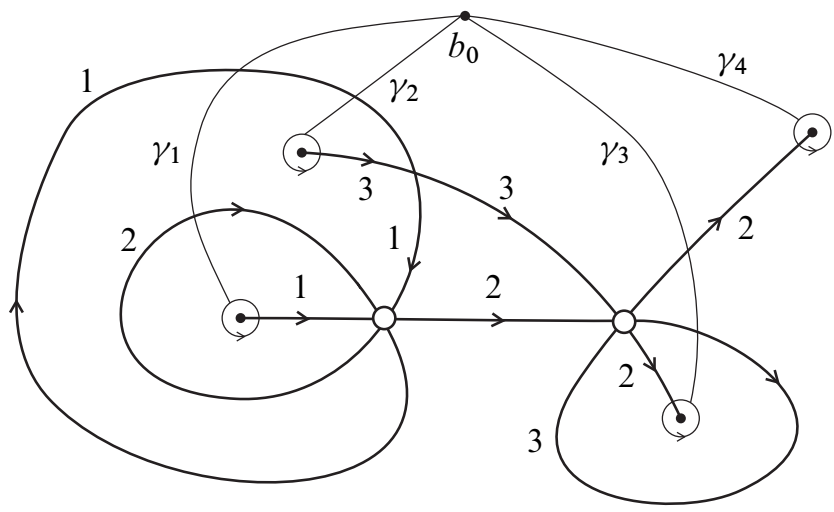

Figure 9: Monodromy of a chart $\Gamma$

Theorem 2.11 Suppose that $g$ is greater than two. Let $f$ and $f^{\prime}$ be Lefschetz fibrations of genus $g$ over $B$, and $\Gamma$ and $\Gamma^{\prime}$ charts corresponding to $f$ and $f^{\prime}$, respectively. Then $f$ is strictly isomorphic to $f^{\prime}$ if and only if $\Gamma$ is transformed to $\Gamma^{\prime}$ by a finite sequence of chart moves of type $W$, chart moves of transitions, chart moves of conjugacy type, and ambient isotopies of $B$ relative to $b_{0}$.

Proposition 2.9 and Theorem 2.11 follow from a classification theorem of Lefschetz fibrations due to Kas [23] and Matsumoto [32] together with fundamental theorems on charts and chart moves by Kamada [20, Sections 4-8].

We end this subsection with a definition and chart description of fiber sums of Lefschetz fibrations. Let $f: M \rightarrow B$ and $f^{\prime}: M^{\prime} \rightarrow B^{\prime}$ be Lefschetz fibrations of genus $g$. Take 
regular values $b_{0} \in B$ and $b_{0}^{\prime} \in B^{\prime}$ of $f$ and $f^{\prime}$, and small disks $D_{0} \subset B-\Delta$ and $D_{0}^{\prime} \subset B-\Delta^{\prime}$ near $b_{0}$ and $b_{0}^{\prime}$, respectively. Consider general fibers $F_{0}:=f^{-1}\left(b_{0}\right)$ and $F_{0}^{\prime}:=f^{\prime-1}\left(b_{0}^{\prime}\right)$ and orientation-preserving diffeomorphisms $\Phi: \Sigma_{g} \rightarrow F_{0}$ and $\Phi^{\prime}: \Sigma_{g} \rightarrow F_{0}^{\prime}$, respectively.

Definition 2.12 Let $\Psi: \Sigma_{g} \rightarrow \Sigma_{g}$ be an orientation-preserving diffeomorphism and $r: \partial D_{0} \rightarrow \partial D_{0}^{\prime}$ an orientation-reversing diffeomorphism. The new manifold $M \#_{F} M^{\prime}$ obtained by gluing $M-f^{-1}\left(\operatorname{Int} D_{0}\right)$ and $M^{\prime}-f^{\prime-1}\left(\operatorname{Int} D_{0}^{\prime}\right)$ by $\left(\Phi^{\prime} \circ \Psi \circ \Phi^{-1}\right) \times r$ admits a Lefschetz fibration $f \#_{\Psi} f^{\prime}: M \#_{F} M^{\prime} \rightarrow B \# B^{\prime}$ of genus $g$. We call $f \#_{\Psi} f^{\prime}$ the fiber sum of $f$ and $f^{\prime}$ with respect to $\Psi$. Although the diffeomorphism type of $M \#_{F} M^{\prime}$ and the isomorphism type of $f \#_{\Psi} f^{\prime}$ depend on a choice of the diffeomorphism $\Psi$ in general, we often abbreviate $f \#_{\Psi} f^{\prime}$ as $f \# f^{\prime}$.

Let $\Gamma$ and $\Gamma^{\prime}$ be charts corresponding to $f$ and $f^{\prime}$, and $D_{0}$ and $D_{0}^{\prime}$ small disks near $b_{0}$ and $b_{0}^{\prime}$ disjoint from $\Gamma$ and $\Gamma^{\prime}$, respectively. Connecting $B-\operatorname{Int} D_{0}$ with $B^{\prime}-$ Int $D_{0}^{\prime}$ by a tube, we have a connected sum $B \# B^{\prime}$ of $B$ and $B^{\prime}$. Let $w$ be a word in $\mathcal{X} \cup \mathcal{X}^{-1}$ which represents the mapping class of $\Psi$ in $\mathcal{M}_{g}$. Let $\Gamma \#_{w} \Gamma^{\prime}$ be the union of $\Gamma, \Gamma^{\prime}$, and hoops on the tube representing $w$ (see Figure 10). Then the fiber sum $f \#_{\Psi} f^{\prime}$ is described by this new chart $\Gamma \#_{w} \Gamma^{\prime}$ in $B \# B^{\prime}$ with base point $b_{0}$. If the word $w$ is trivial, then the chart $\Gamma \#_{w} \Gamma^{\prime}$ is denoted also by $\Gamma \oplus \Gamma^{\prime}$, which is called a product of $\Gamma$ and $\Gamma^{\prime}$.

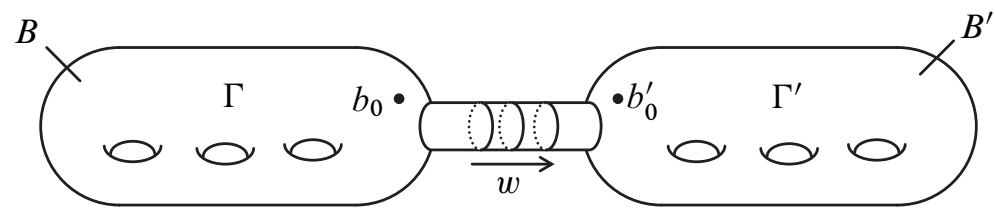

Figure 10: Chart $\Gamma \#_{w} \Gamma^{\prime}$ in $B \# B^{\prime}$

\section{Signature of Lefschetz fibrations}

In this section we review the signature cocycle discovered by Meyer and prove a signature theorem for Lefschetz fibrations.

\subsection{Meyer's signature cocycle}

In this subsection we give a brief survey on Meyer's signature cocycle. We begin with the definition of the signature cocycle. Let $g$ be a positive integer. 
Definition 3.1 (Meyer [33]) For $A, B \in \mathrm{Sp}(2 g, \mathbb{Z})$, we consider the vector space

$$
V_{A, B}:=\left\{(x, y) \in \mathbb{R}^{2 g} \times \mathbb{R}^{2 g} \mid\left(A^{-1}-I_{2 g}\right) x+\left(B-I_{2 g}\right) y=0\right\}
$$

and the bilinear form $\langle,\rangle_{A, B}: V_{A, B} \times V_{A, B} \rightarrow \mathbb{R}$ defined by

$$
\left\langle\left(x_{1}, y_{1}\right),\left(x_{2}, y_{2}\right)\right\rangle_{A, B}:=\left(x_{1}+y_{1}\right) \cdot J\left(I_{2 g}-B\right) y_{2},
$$

where $\cdot$ is the standard inner product of $\mathbb{R}^{2 g}$ and $J=\left(\begin{array}{cc}0 & I_{g} \\ -I_{g} & 0\end{array}\right)$. Since $\langle,\rangle_{A, B}$ is symmetric, we can define an integer $\tau_{g}(A, B)$ to be the signature of $\left(V_{A, B},\langle,\rangle_{A, B}\right)$. The map $\tau_{g}: \operatorname{Sp}(2 g, \mathbb{Z}) \times \operatorname{Sp}(2 g, \mathbb{Z}) \rightarrow \mathbb{Z}$ is called the signature cocycle.

Let $P$ be a compact connected oriented surface of genus 0 with three boundary components and $\pi: E \rightarrow P$ a fiber bundle over $P$ with fiber $\Sigma_{g}$ and structure group Diff $+\Sigma_{g}$. The fundamental group $\pi_{1}(P, *)$ of $P$ with base point $*$ is a free group generated by two loops $a$ and $b$ depicted in Figure 11. If we take an orientationpreserving diffeomorphism $\Sigma_{g} \rightarrow \pi^{-1}(*)$, we obtain the monodromy representation $\pi_{1}(P, *) \rightarrow \mathcal{M}_{g}$ which sends $a$ to $\alpha$ and $b$ to $\beta$. Since $\mathcal{M}_{g}^{*}$ acts on $H:=H_{1}\left(\Sigma_{g} ; \mathbb{Z}\right)$ and preserves the intersection form, we have a representation $\mathcal{M}_{g}^{*} \rightarrow \operatorname{Sp}(2 g, \mathbb{Z})$ by fixing a symplectic basis on $H$. Let $A$ and $B$ denote matrices corresponding to $\alpha$ and $\beta$, respectively.

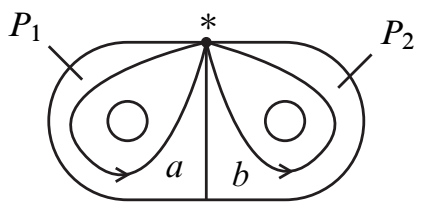

Figure 11: Pair of pants $P$

Meyer closely studied the signature of the total space $E$ to obtain the following theorem.

Theorem 3.2 (Meyer [33]) The signature $\sigma(E)$ of $E$ is equal to $-\tau_{g}(A, B)$.

Theorem 3.2 and Novikov's additivity implies that $\tau_{g}$ is a 2 -cocycle of $\operatorname{Sp}(2 g, \mathbb{Z})$.

We recall the Maslov index of a triple of Lagrangian subspaces and Wall's non-additivity theorem, which are used in the proof of Theorem 3.2.

Let $V$ be a real vector space of dimension $2 n, \omega \in \Lambda^{2} V^{*}$ a symplectic form on $V$, and $\Lambda(V, \omega)$ the Lagrangian Grassmannian of $(V, \omega)$, which is the set of Lagrangian subspaces of $(V, \omega)$. For $L_{1}, L_{2}, L_{3} \in \Lambda(V, \omega)$, the bilinear form

$$
\Psi:\left(L_{3}+L_{1}\right) \cap L_{2} \times\left(L_{3}+L_{1}\right) \cap L_{2} \rightarrow \mathbb{R}, \quad(v, w) \mapsto \omega\left(v, w_{3}\right),
$$


where $v, w \in\left(L_{3}+L_{1}\right) \cap L_{2}$ and $w=w_{1}+w_{3}\left(w_{1} \in L_{1}, w_{3} \in L_{3}\right)$, is symmetric. We define an integer $i\left(L_{1}, L_{2}, L_{3}\right)$ to be the signature of $\left(\left(L_{3}+L_{1}\right) \cap L_{2}, \Psi\right)$, which is called the ternary Maslov index of the triple $\left(L_{1}, L_{2}, L_{3}\right)$.

Let $M_{1}, M_{2}$ be compact oriented smooth 4-manifolds, $X_{1}, X_{2}, X_{3}$ compact oriented smooth 3-manifolds, and $\Sigma$ a closed oriented smooth 2-manifold. We assume that $M=M_{1} \cup M_{2}, \partial M_{1}=X_{1} \cup X_{2}, \partial M_{2}=X_{2} \cup X_{3}, \partial X_{1}=\partial X_{2}=\partial X_{3}=\Sigma$, and the orientations of these manifolds satisfy

$$
\begin{gathered}
{[M]=\left[M_{1}\right]+\left[M_{2}\right], \quad \partial_{*}\left[M_{1}\right]=\left[X_{2}\right]-\left[X_{1}\right], \quad \partial_{*}\left[M_{2}\right]=\left[X_{3}\right]-\left[X_{2}\right],} \\
\partial_{*}\left[X_{1}\right]=\partial_{*}\left[X_{2}\right]=\partial_{*}\left[X_{3}\right]=[\Sigma] .
\end{gathered}
$$

Let $\omega: V \times V \rightarrow \mathbb{R}$ be the intersection form on $V:=H_{1}(\Sigma ; \mathbb{R})$ and $L_{i}$ the kernel of the homomorphism $V \rightarrow H_{1}\left(X_{i} ; \mathbb{R}\right)$ induced by the inclusion $\Sigma \rightarrow X_{i}$ for $i=$ 1,2,3. Since $L_{i} \in \Lambda(V, \omega)$ for $i=1,2,3$, we can define the ternary Maslov index $i\left(L_{1}, L_{2}, L_{3}\right)$ of the triple $\left(L_{1}, L_{2}, L_{3}\right)$.

Theorem 3.3 (Wall [42]) $\sigma(M)=\sigma\left(M_{1}\right)+\sigma\left(M_{2}\right)-i\left(L_{1}, L_{2}, L_{3}\right)$.

Gambaudo and Ghys [10] (and independently the first author) made use of Theorem 3.3 to give the following proof of Theorem 3.2. See also Gilmer and Masbaum [12].

Proof of Theorem 3.2 Consider $P$ to be a boundary sum of two annuli $P_{1}$ and $P_{2}$ (see Figure 11). We set $M:=E, M_{i}:=\pi^{-1}\left(P_{i}\right)(i=1,2), X_{2}:=M_{1} \cap M_{2}$, $X_{1}:=\partial M_{1}-$ Int $X_{2}, X_{3}:=\partial M_{3}-$ Int $X_{2}$, and $\Sigma:=\partial X_{2}$. Applying Theorem 3.3 to these manifolds, we have

$$
\sigma(E)=\sigma\left(M_{1}\right)+\sigma\left(M_{2}\right)-i\left(L_{1}, L_{2}, L_{3}\right)=-i\left(L_{1}, L_{2}, L_{3}\right)
$$

because each of $M_{1}$ and $M_{2}$ is a product of a mapping torus with an interval, which has signature zero. Since the bordered component of $X_{i}$ is diffeomorphic to $I \times \Sigma_{g}$ for $i=1,2,3$, we put $V:=H \oplus H, \omega:=\mu \oplus(-\mu)$, and obtain

$$
\begin{aligned}
& L_{1}=\left\{\left(-\xi, \alpha_{*}^{-1}(\xi)\right) \in V \mid \xi \in H\right\}, \\
& L_{2}=\{(-\xi, \xi) \in V \mid \xi \in H\}, \\
& L_{3}=\left\{\left(-\xi, \beta_{*}(\xi)\right) \in V \mid \xi \in H\right\},
\end{aligned}
$$

where $H$ is the first homology $H_{1}\left(\Sigma_{g} ; \mathbb{R}\right)$ of $\Sigma_{g}$ and $\mu: H \times H \rightarrow \mathbb{R}$ is the intersection form of $\Sigma_{g}$. It is easily seen that the subspace $\left(L_{1}+L_{3}\right) \cap L_{2}$ is written as

$$
\left(L_{1}+L_{3}\right) \cap L_{2}=\left\{\left(-\xi-\eta, \alpha_{*}^{-1}(\xi)+\beta_{*}(\eta)\right) \in V \mid \xi+\eta=\alpha_{*}^{-1}(\xi)+\beta_{*}(\eta)(\xi, \eta \in H)\right\}
$$


and the symmetric bilinear form $\Psi$ on $\left(L_{1}+L_{3}\right) \cap L_{2}$ is written as $\Psi\left(\left(-\xi-\eta, \alpha_{*}^{-1}(\xi)+\beta_{*}(\eta)\right),\left(-\xi^{\prime}-\eta^{\prime}, \alpha_{*}^{-1}\left(\xi^{\prime}\right)+\beta_{*}\left(\eta^{\prime}\right)\right)\right)=\mu\left(\xi+\eta,\left(\mathrm{id}-\beta_{*}\right)\left(\eta^{\prime}\right)\right)$.

We consider the vector space

$$
U_{\alpha, \beta}:=\left\{(\xi, \eta) \in V \mid\left(\alpha_{*}^{-1}-\mathrm{id}\right)(\xi)+\left(\beta_{*}-\mathrm{id}\right)(\eta)=0\right\}
$$

and the symmetric bilinear form $\langle,\rangle_{\alpha, \beta}$ on $U_{\alpha, \beta}$ defined by

$$
\left\langle(\xi, \eta),\left(\xi^{\prime}, \eta^{\prime}\right)\right\rangle_{\alpha, \beta}:=\mu\left(\xi+\eta,\left(\mathrm{id}-\beta_{*}\right)\left(\eta^{\prime}\right)\right) \quad\left((\xi, \eta),\left(\xi^{\prime}, \eta^{\prime}\right) \in U_{\alpha, \beta}\right) .
$$

Since the linear map $U_{\alpha, \beta} \rightarrow\left(L_{1}+L_{3}\right) \cap L_{2}:(\xi, \eta) \mapsto(-\xi-\eta, \xi+\eta)$ is compatible with the bilinear forms, the signature of $\left(\left(L_{1}+L_{3}\right) \cap L_{2}, \Psi\right)$ is equal to that of $\left(U_{\alpha, \beta},\langle,\rangle_{\alpha, \beta}\right)$, which is isomorphic to $\left(V_{A, B},\langle,\rangle_{A, B}\right)$ under a choice of a symplectic basis of $H$. Therefore we conclude that $i\left(L_{1}, L_{2}, L_{3}\right)=\tau_{g}(A, B)$.

Remark 3.4 It is known that $\tau_{g}$ is a normalized, symmetric 2-cocycle of $\operatorname{Sp}(2 g, \mathbb{Z})$ and invariant under conjugation. The cohomology class $\left[\tau_{g}\right] \in H^{2}(\operatorname{Sp}(2 g, \mathbb{Z}) ; \mathbb{Z})$ corresponds to $-4 c_{1}$ under the homomorphisms

$$
H^{2}(\operatorname{Sp}(2 g, \mathbb{Z}) ; \mathbb{Z}) \leftarrow H^{2}(B \operatorname{Sp}(2 g, \mathbb{R}) ; \mathbb{Z}) \cong H^{2}(B U(g) ; \mathbb{Z}) \cong \mathbb{Z} .
$$

For more details, see Meyer [33], Turaev [39], Barge and Ghys [3], and Kuno [27].

\subsection{A signature formula}

In this subsection we describe the signature of a Lefschetz fibration of genus greater than two in terms of charts. Let $g$ be an integer greater than two.

Let $B$ be a connected closed oriented surface and $\Gamma$ a chart in $B$. We denote the number of white vertices of type $r_{F}(i, j)$ (resp. $\left.r_{B}(i), r_{C}, r_{L}, r_{H}\right)$ minus the number of white vertices of type $r_{F}(i, j)^{-1}$ (resp. $\left.r_{B}(i)^{-1}, r_{C}^{-1}, r_{L}^{-1}, r_{H}^{-1}\right)$ included in $\Gamma$ by $n_{F}(i, j)(\Gamma)$ (resp. $\left.n_{B}(i)(\Gamma), n_{C}(\Gamma), n_{L}(\Gamma), n_{H}(\Gamma)\right)$. Similarly, we denote the number of black vertices of type $\ell_{0}(i)^{ \pm 1}$ (resp. $\ell_{h}^{ \pm 1}$ ) included in $\Gamma$ by $n_{0}^{ \pm}(i)(\Gamma)$ (resp. $\left.n_{h}^{ \pm}(\Gamma)\right)$, and set $n_{0}(i)(\Gamma):=n_{0}^{+}(i)(\Gamma)-n_{0}^{-}(i)(\Gamma)\left(\operatorname{resp} . n_{h}(\Gamma):=n_{h}^{+}(\Gamma)-n_{h}^{-}(\Gamma)\right)$ and $n_{0}^{ \pm}(\Gamma):=\sum_{i=0}^{2 g} n_{0}^{ \pm}(i)(\Gamma)$.

Definition 3.5 The number

is called the signature of $\Gamma$.

$$
\sigma(\Gamma):=-6 n_{C}(\Gamma)-n_{L}(\Gamma)+\sum_{h=1}^{[g / 2]}(4 h(h+1)-1) n_{h}(\Gamma)
$$


Let $f: M \rightarrow B$ be a Lefschetz fibration of genus $g$ and $\Gamma$ a chart in $B$ corresponding to $f$. The purpose of this subsection is to show the following theorem.

Theorem 3.6 The signature $\sigma(M)$ of $M$ is equal to $\sigma(\Gamma)$.

Remark 3.7 It immediately follows from Theorem 3.6 that $\sigma(\Gamma)$ is invariant under chart moves of type $\mathrm{W}$ and chart moves of transition. Although any combinatorial proof of this fact does not seem to be known, Hasegawa [15] proved that $\sigma(\Gamma)$ is invariant under chart moves of transitions by a purely combinatorial method on the assumption that it is invariant under chart moves of type $\mathrm{W}$.

Let $\tilde{\mathcal{X}}$ be the set of right-handed Dehn twists along simple closed curves in $\Sigma_{g}$ and $\widetilde{\mathcal{R}}$ the set of words in $\widetilde{\mathcal{X}} \cup \tilde{\mathcal{X}}^{-1}$ representing an element of the kernel of the natural epimorphism from the free group generated by $\tilde{\mathcal{X}}$ to $\mathcal{M}_{g}$.

Definition 3.8 For a word $w=\alpha_{1} \cdots \alpha_{n} \in \widetilde{\mathcal{R}}$, we define an integer

$$
I_{g}(w):=-\sum_{j=1}^{n-1} \tau_{g}\left(\bar{\alpha}_{n-j}, \bar{\alpha}_{n-j+1} \cdots \bar{\alpha}_{n}\right)-s(w),
$$

where $\tau_{g}$ is the signature cocycle (Definition 3.1), $\bar{\alpha}$ is the image of $\alpha \in \tilde{\mathcal{X}} \cup \tilde{\mathcal{X}}^{-1}$ under the composition of the natural map $\tilde{\mathcal{X}} \cup \tilde{\mathcal{X}}^{-1} \rightarrow \mathcal{M}_{g}$ and a natural epimorphism $\mathcal{M}_{g}^{*} \rightarrow \operatorname{Sp}(2 g, \mathbb{Z})$, and $s(w)$ is the number of Dehn twists along separating simple closed curves included in $w$.

Suppose that $B$ is a 2 -sphere. If we choose a monodromy representation $\rho$ and a Hurwitz arc system $\mathcal{A}$ for $\Delta$ with base point $b_{0}$, we have a Hurwitz system $\left(\alpha_{1}, \ldots, \alpha_{n}\right) \in\left(\mathcal{M}_{g}\right)^{n}$ of $f$. Since $\alpha_{1}, \ldots, \alpha_{n}$ are Dehn twists and $\alpha_{1} \cdots \alpha_{n}=1$ in $\mathcal{M}_{g}$, we think $\left(\alpha_{1}, \ldots, \alpha_{n}\right)$ as a word $w:=\alpha_{1} \cdots \alpha_{n}$ in $\widetilde{\mathcal{R}}$. Theorem 3.2 and Novikov's additivity for signature imply the next theorem.

Theorem 3.9 (Endo and Nagami [8]) The signature $\sigma(M)$ of $M$ is equal to $I_{g}(w)$.

We are now ready to prove Theorem 3.6.

Proof of Theorem 3.6 Choose a base point $b_{0} \in B-\Gamma$ and a disk $D$ in $B-\Gamma$ centered at $b_{0}$. We denote the set of edges of $\Gamma$ by $E(\Gamma)$. For each $e \in E(\Gamma)$, we choose a point $b_{e}$ in a region of $B-\Gamma$ adjacent to $e$, and a simple path $\gamma_{e}$ from $b_{e}$ to $b_{0}$ which intersects with edges of $\Gamma$ transversely and does not intersect with vertices of $\Gamma$. Let $w_{e}$ be the intersection word of $\gamma_{e}$ with respect to $\Gamma$ and $i_{e} \in\{0,1, \ldots, 2 g\}$ 


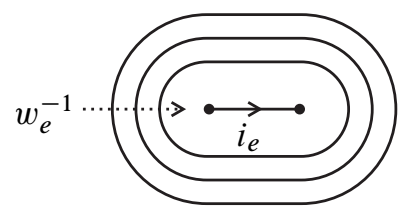

Figure 12: Chart $\Gamma_{e}$

the label of $e$. We choose a family $\left\{D_{e}\right\}_{e \in E(\Gamma)}$ of mutually disjoint disks included in $D$ and put the chart $\Gamma_{e}$ depicted in Figure 12 in $D_{e}$ for each $e$.

Taking the union of $\Gamma$ with $\Gamma_{e}$ for all $e \in E(\Gamma)$, we obtain a new chart $\Gamma_{1}$ in $B$, which describes a fiber sum $f_{1}: M_{1} \rightarrow B$ of $f$ with Lefschetz fibrations over $S^{2}$ described by a free edge. For each $e \in E(\Gamma)$, we apply channel changes as in Figure 13 to let a free edge pass through the edges intersecting with $\gamma_{e}$. We then apply a channel change as in Figure 14 to "cut" $e$ into two edges. Thus we obtain a new chart $\Gamma_{2}$ in $B$.
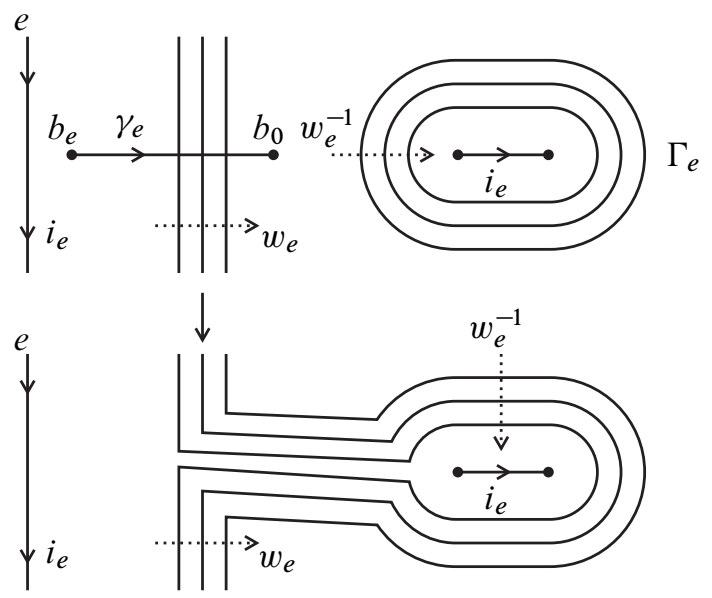

Figure 13: Channel change

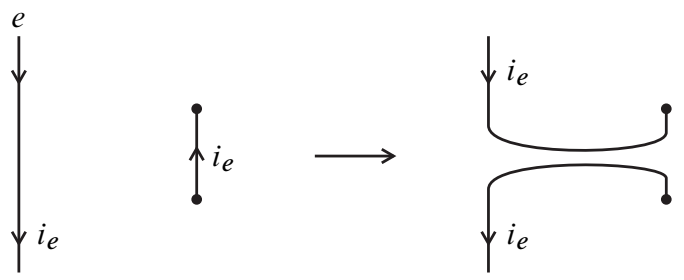

Figure 14: Channel change

Since each component of $\Gamma_{2}$ is a tree, a Lefschetz fibration $f_{2}: M_{2} \rightarrow B$ corresponding to $\Gamma_{2}$ is a fiber sum of a Lefschetz fibration $f_{3}: M_{3} \rightarrow S^{2}$ with a trivial $\Sigma_{g}$-bundle 
over $B$. Drawing a copy of $\Gamma_{2}$ in $S^{2}$, we have a chart $\Gamma_{3}$ corresponding to $f_{3}$. The signature of a Lefschetz fibration over $S^{2}$ described by a free edge is equal to zero because $\tau_{g}\left(A, A^{-1}\right)=0$ for any $A \in \operatorname{Sp}(2 g, \mathbb{Z})$ (see Meyer [33, Section 2]). Hence we have

$$
\sigma(M)=\sigma\left(M_{1}\right)=\sigma\left(M_{2}\right)=\sigma\left(M_{3}\right)+\sigma\left(\Sigma_{g} \times B\right)=\sigma\left(M_{3}\right)
$$

by Theorem 2.11 and Novikov's additivity. Since we did not change the numbers of white vertices and black vertices of type $\ell_{h}^{ \pm}$to make $\Gamma_{3}$ from $\Gamma$, we see $\sigma\left(\Gamma_{3}\right)=\sigma(\Gamma)$. Hence we only have to show $\sigma\left(M_{3}\right)=\sigma\left(\Gamma_{3}\right)$ in order to conclude $\sigma(M)=\sigma(\Gamma)$.

Applying chart moves of transition to each component of $\Gamma_{3}$ as in Figure 15, we remove white vertices of type $r_{F}(i, j)^{ \pm 1}, r_{B}(i)^{ \pm 1}, r_{H}^{ \pm 1}$ to obtain a union of copies of $L_{0}(i), L_{h}, L_{h}^{*}, R_{C}, R_{C}^{*}, R_{L}, R_{L}^{*}$, where $L_{0}(i), L_{h}, R_{C}, R_{L}$ are charts depicted in Figure 16 and Figure 17, and $L_{h}^{*}$ (resp. $R_{C}^{*}, R_{L}^{*}$ ) is the mirror image of $L_{h}$ (resp. $R_{C}, R_{L}$ ) with edges orientation reversed. For the proof of $\sigma\left(M_{3}\right)=\sigma\left(\Gamma_{3}\right)$, it is enough to show that the signature of a Lefschetz fibration described by each of these charts coincides with the signature of the chart.

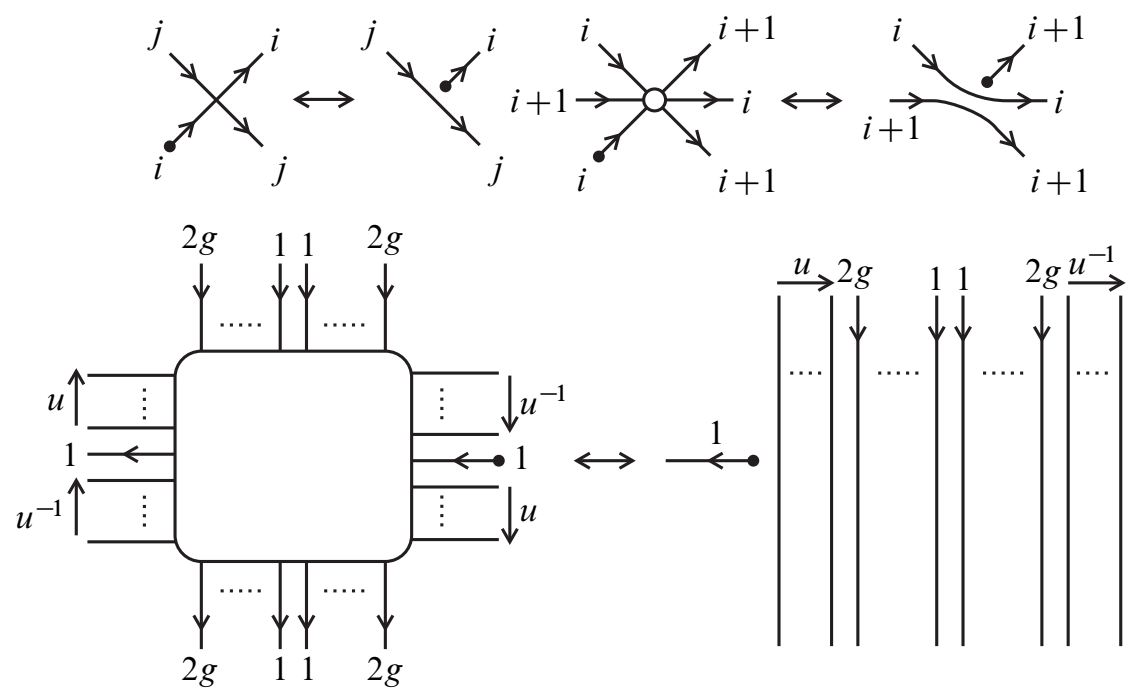

Figure 15: Chart moves of transition
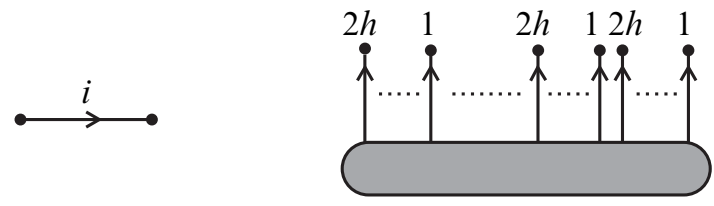

Figure 16: Charts $L_{0}(i)$ and $L_{h}$ 

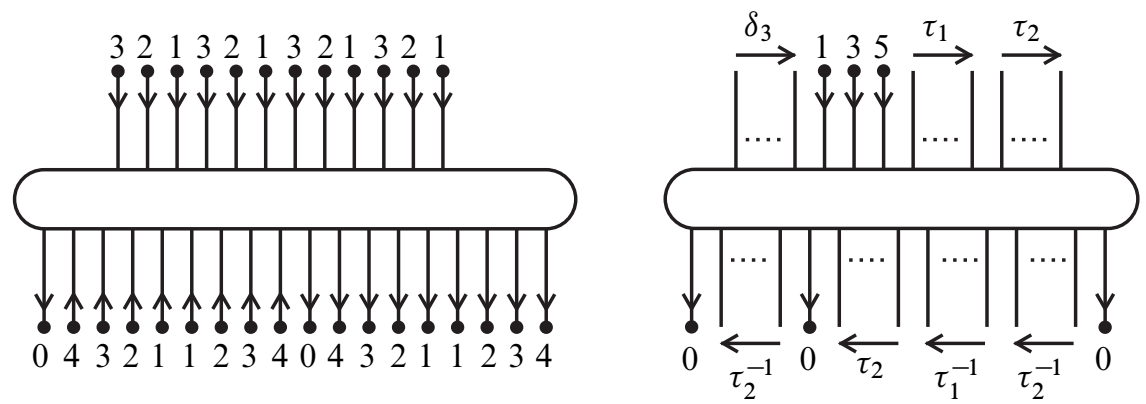

Figure 17: Charts $R_{C}$ and $R_{L}$

Let $\Gamma_{4}$ be one of $L_{0}(i), L_{h}, L_{h}^{*}, R_{C}, R_{C}^{*}, R_{L}, R_{L}^{*}$ drawn in $S^{2}$ and $f_{4}: M_{4} \rightarrow S^{2}$ a Lefschetz fibration described by $\Gamma_{4}$. If $\Gamma_{4}$ is equal to $L_{0}(i)$, it is easily seen that $\sigma\left(M_{4}\right)=\sigma\left(\Gamma_{4}\right)$. If $\Gamma_{4}$ is equal to $L_{h}$, the word $\ell_{h}^{-1} \sigma_{h}$ corresponds to a Hurwitz system of $f_{4}$ (see Figure 16), where $\sigma_{h}$ is a right-handed Dehn twist along the curve $s_{h}$ depicted in Figure 1. Thus we have

$$
\sigma\left(M_{4}\right)=I_{g}\left(\ell_{h}^{-1} \sigma_{h}\right)=4 h(h+1)-1=\sigma\left(\Gamma_{4}\right)
$$

from Definition 3.5, Theorem 3.9, and explicit computations for $I_{g}$ due to Endo and Nagami [8, Lemma 3.5, Proposition 3.9]. If $\Gamma_{4}$ is equal to $R_{C}$ (resp. $R_{L}$ ), the word $r_{C}$ (resp. $r_{L}$ ) corresponds to a Hurwitz system of $f_{4}$ (see Figure 17). Thus we have

$$
\sigma\left(M_{4}\right)=I_{g}\left(r_{C}\right)=-6=\sigma\left(\Gamma_{4}\right) \quad\left(\text { resp. } \sigma\left(M_{4}\right)=I_{g}\left(r_{L}\right)=-1=\sigma\left(\Gamma_{4}\right)\right)
$$

from Definition 3.5, Theorem 3.9, and formulas of Endo and Nagami [8, Lemma 3.5, Remark 3.7, Propositions 3.9 and 3.10]. Suppose that $\Gamma_{4}$ is equal to one of $L_{h}^{*}, R_{C}^{*}, R_{L}^{*}$. The mirror image $\Gamma_{4}^{*}$ of $\Gamma_{4}$ with edges orientation reversed corresponds to the Lefschetz fibration $f_{4}:-M_{4} \rightarrow S^{2}$ with total space orientation reversed. Hence we have

$$
\sigma\left(M_{4}\right)=-\sigma\left(-M_{4}\right)=-\sigma\left(\Gamma_{4}^{*}\right)=\sigma\left(\Gamma_{4}\right)
$$

because we have already shown that $\sigma\left(M_{4}\right)=\sigma\left(\Gamma_{4}\right)$ is valid for $\Gamma_{4}=L_{h}, R_{C}, R_{L}$. This completes the proof of Theorem 3.6.

\section{Stabilization theorems}

In this section we prove two theorems on stabilization of Lefschetz fibrations under taking fiber sums with copies of a fixed Lefschetz fibration.

Following Auroux [2], we first introduce a notion of universality for Lefschetz fibrations. Suppose that $g$ is greater than two. 
Definition 4.1 A Lefschetz fibration of genus $g$ over $S^{2}$ is called universal if it is irreducible, chiral, and it contains $2 g+1$ singular fibers of type $\mathrm{I}^{+}$whose vanishing cycles $a_{0}, a_{1}, \ldots, a_{2 g} \subset \Sigma_{g}$ satisfy the following conditions:

(i) $a_{i}$ and $a_{i+1}$ intersect transversely at one point for every $i \in\{1, \ldots, 2 g-1\}$.

(ii) $a_{0}$ and $a_{4}$ intersect transversely at one point.

(iii) $a_{i}$ and $a_{j}$ does not intersect for other pairs $(i, j)$.

A Lefschetz fibration over $S^{2}$ is universal if and only if it is described by a chart $\Gamma_{0}$ depicted in Figure 18 by virtue of Proposition 2.9, where the blank labeled by $\mathrm{T}_{0}$ is filled only with edges, white vertices, and black vertices of type $\ell_{0}(i)$.

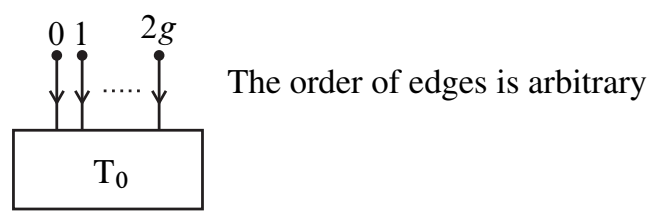

Figure 18: Universal chart $\Gamma_{0}$

Remark 4.2 A universal Lefschetz fibration exists for every $g$ greater than two. For example, the Lefschetz fibrations $f_{g}^{0}, f_{g}^{A}, f_{g}^{B}, f_{g}^{C}, f_{g}^{D}$ constructed by Auroux [2] are universal except $f_{g}^{D}$ for $g=3$. There are many universal Lefschetz fibrations of genus $g$ for a fixed $g$.

We now state the first of our main theorems. Let $B$ be a connected closed oriented surface and $f_{0}: M_{0} \rightarrow S^{2}$ a universal Lefschetz fibration of genus $g$.

Theorem 4.3 Let $f: M \rightarrow B$ and $f^{\prime}: M^{\prime} \rightarrow B$ be Lefschetz fibrations of genus $g$. There exists a non-negative integer $N$ such that $f \# N f_{0}$ is isomorphic to $f^{\prime} \# N f_{0}$ if and only if the following conditions hold:

(i) $n_{0}^{ \pm}(f)=n_{0}^{ \pm}\left(f^{\prime}\right)$.

(ii) $n_{h}^{ \pm}(f)=n_{h}^{ \pm}\left(f^{\prime}\right)$ for every $h=1, \ldots,[g / 2]$.

(iii) $\sigma(M)=\sigma\left(M^{\prime}\right)$.

Remark 4.4 Auroux [2] proved the "if" part of Theorem 4.3 for chiral Lefschetz fibrations over $S^{2}$ under the assumption that $f$ and $f^{\prime}$ have sections with the same self-intersection number. Hasegawa [15] gave another proof of Auroux's theorem by using chart descriptions. Moreover, he removed the assumption on existence and self-intersection number of sections in Auroux's theorem. 
Remark 4.5 The isomorphism class of a fiber sum $f \#_{\Psi} f_{0}$ of a Lefschetz fibration $f$ with a universal Lefschetz fibration $f_{0}$ does not depend on a choice of an orientationpreserving diffeomorphism $\Psi$ (see the proof of Theorem 4.3).

Proof of Theorem 4.3 We first prove the "if" part. Assume that $f$ and $f^{\prime}$ satisfy the conditions (i), (ii), and (iii). Let $\Gamma$ and $\Gamma^{\prime}$ be charts in $B$ corresponding to $f$ and $f^{\prime}$, respectively. We suppose that $f_{0}$ is described by a chart $\Gamma_{0}$ depicted in Figure 18. Since every edge has two adjacent vertices, the sum of the signed numbers of adjacent edges for all vertices of $\Gamma$ is equal to zero:

$$
10 n_{C}(\Gamma)+n_{L}(\Gamma)-\sum_{i=0}^{2 g} n_{0}(i)(\Gamma)-4 \sum_{h=1}^{[g / 2]} h(2 h+1) \cdot n_{h}(\Gamma)=0 .
$$

A similar equality for $\Gamma^{\prime}$ also holds. Interpreting the conditions (i) and (ii) as conditions on $\Gamma$ and $\Gamma^{\prime}$, we have $\sum_{i=0}^{2 g} n_{0}(i)(\Gamma)=\sum_{i=0}^{2 g} n_{0}(i)\left(\Gamma^{\prime}\right)$ and $n_{h}(\Gamma)=n_{h}\left(\Gamma^{\prime}\right)$ for $h=1, \ldots,[g / 2]$. Thus we obtain

$$
10 n_{C}(\Gamma)+n_{L}(\Gamma)=10 n_{C}\left(\Gamma^{\prime}\right)+n_{L}\left(\Gamma^{\prime}\right) .
$$

On the other hand, we have

$$
-6 n_{C}(\Gamma)-n_{L}(\Gamma)=-6 n_{C}\left(\Gamma^{\prime}\right)-n_{L}\left(\Gamma^{\prime}\right)
$$

by the condition (iii), Theorem 3.6, and $n_{h}(\Gamma)=n_{h}\left(\Gamma^{\prime}\right)$ for $h=1, \ldots,[g / 2]$. Hence $n_{C}(\Gamma)=n_{C}\left(\Gamma^{\prime}\right)$ and $n_{L}(\Gamma)=n_{L}\left(\Gamma^{\prime}\right)$.

Let $N$ be an integer larger than both of the number of edges of $\Gamma$ and that of $\Gamma^{\prime}$. Choose a base point $b_{0} \in B-\left(\Gamma \cup \Gamma^{\prime}\right)$. The fiber sum $f \# N f_{0}$ is described by a chart $\left(\cdots\left(\left(\Gamma \#_{w_{1}} \Gamma_{0}\right) \#_{w_{2}} \Gamma_{0}\right) \cdots\right) \#_{w_{N}} \Gamma_{0}$ for some words $w_{1}, \ldots, w_{N}$ in $\mathcal{X} \cup \mathcal{X}^{-1}$. Since hoops surrounding $\Gamma_{0}$ can be removed by use of the edges of $\Gamma_{0}$ as in Figure 19, the chart is transformed into a product $\Gamma \oplus N \Gamma_{0}$ by channel changes. Similarly, the fiber sum $f^{\prime} \# N f_{0}$ is described by a product $\Gamma^{\prime} \oplus N \Gamma_{0}$.

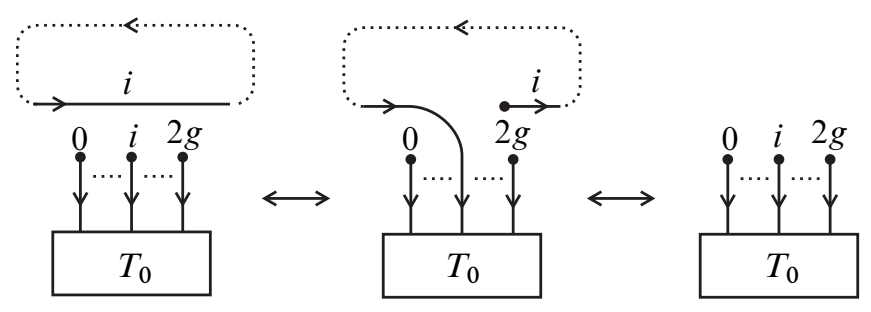

Figure 19: Removing a hoop 
We choose and fix $2 g+1$ edges of $\Gamma_{0}$ which are labeled with $0,1, \ldots, 2 g$ and adjacent to black vertices. We apply chart moves only to these edges in the following. Since $\Gamma_{0}$ can pass through any edge of $\Gamma$ as shown in Figure 20, we can move $\Gamma_{0}$ to any region of $B-\Gamma$ by channel changes.

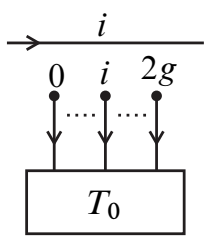

(a)

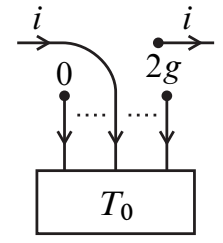

(b)

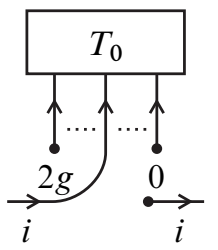

(c)

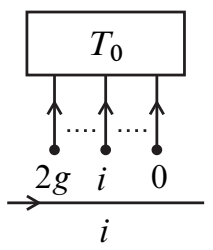

(d)

Figure 20: Passing through an edge

For each edge of $\Gamma$, we move a copy of $\Gamma_{0}$ to a region adjacent to the edge and apply a channel change to the edge and $\Gamma_{0}$ as in Figure 20(a) and (b). Applying chart moves of transition to each component of the chart as in Figure 15, we remove white vertices of type $r_{F}(i, j)^{ \pm 1}, r_{B}(i)^{ \pm 1}, r_{H}^{ \pm 1}$ to obtain a union of copies of $L_{0}(i), \widetilde{L}_{h}, L_{h}^{*}, \widetilde{R}_{C}, \widehat{R}_{C}, \widetilde{R}_{L}, \widehat{R}_{L}, \Gamma_{0}$ shown in Figures $21-23$, where we use a simplification of diagrams as in Figure 24.

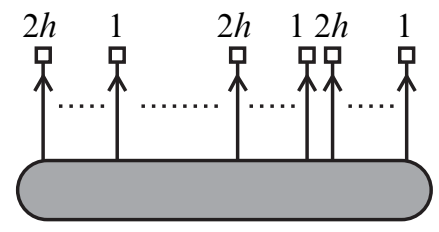

Figure 21: Chart $\widetilde{L}_{h}$
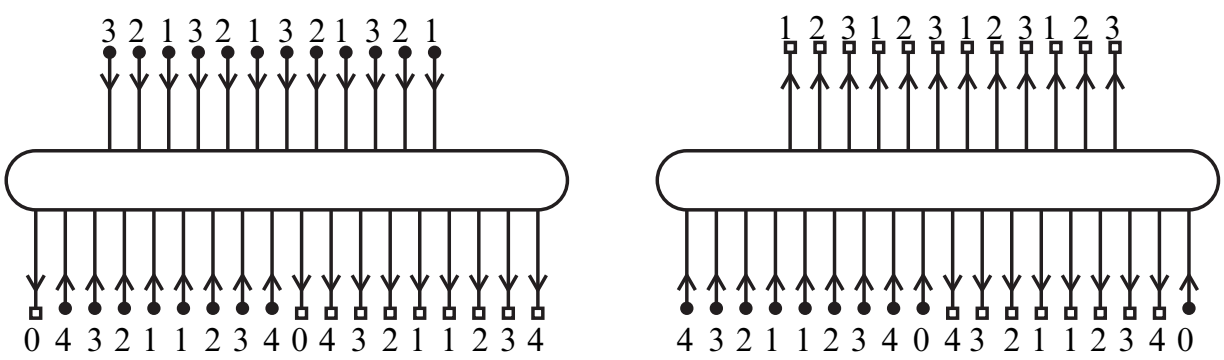

Figure 22: Charts $\widetilde{R}_{C}$ and $\widehat{R}_{C}$ 

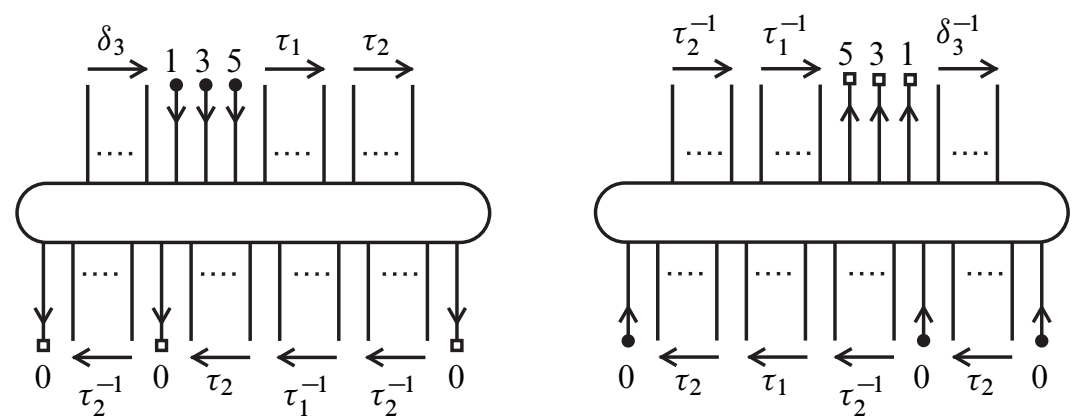

Figure 23: Charts $\widetilde{R}_{L}$ and $\widehat{R}_{L}$

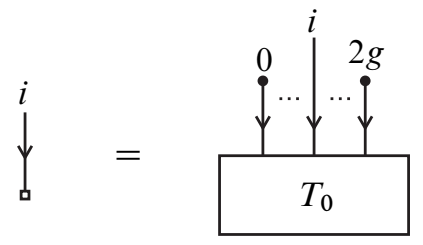

Figure 24: Simplification of diagram

If there is a pair of $\widetilde{R}_{C}$ and $\widehat{R}_{C}$, we remove them by a death of a pair of white vertices to obtain many copies of $\Gamma_{0}$. Similarly, we remove a pair of $\widetilde{R}_{L}$ and $\widehat{R}_{L}$. Since there is at least one $\Gamma_{0}$, any copy of $L_{0}(i)$ can be transformed into $L_{0}(1)$ as in Figure 25.
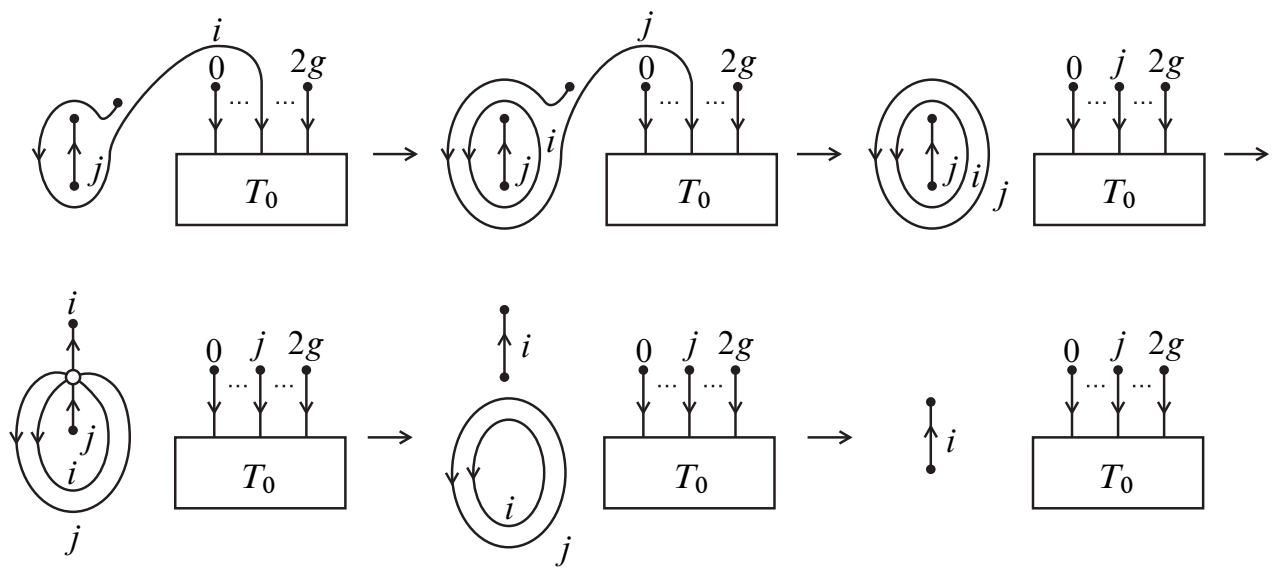

Figure 25: Changing a label $(j=i+1$ or $(i, j)=(4,0))$

Thus we have a union $\Gamma_{1}$ of $n_{0}^{-}(\Gamma)$ copies of $L_{0}(1), n_{h}^{+}(\Gamma)$ copies of $\tilde{L}_{h}, n_{h}^{-}(\Gamma)$ copies of $L_{h}^{*},\left|n_{C}(\Gamma)\right|$ copies of $\widetilde{R}_{C}$ (or $\widehat{R}_{C}$ ), $\left|n_{L}(\Gamma)\right|$ copies of $\widetilde{R}_{L}$ (or $\widehat{R}_{L}$ ), and $k$ copies of $\Gamma_{0}$ for some $k$. A similar argument implies that $\Gamma^{\prime} \oplus N \Gamma_{0}$ is transformed into a union $\Gamma_{1}^{\prime}$ of $n_{0}^{-}\left(\Gamma^{\prime}\right)$ copies of $L_{0}(1), n_{h}^{+}\left(\Gamma^{\prime}\right)$ copies of $\widetilde{L}_{h}, n_{h}^{-}\left(\Gamma^{\prime}\right)$ copies of $L_{h}^{*},\left|n_{C}\left(\Gamma^{\prime}\right)\right|$ copies of $\widetilde{R}_{C}\left(\right.$ or $\left.\hat{R}_{C}\right),\left|n_{L}\left(\Gamma^{\prime}\right)\right|$ copies of $\widetilde{R}_{L}\left(\right.$ or $\left.\hat{R}_{L}\right)$, and $k^{\prime}$ 
copies of $\Gamma_{0}$ for some $k^{\prime}$ by chart moves of type $\mathrm{W}$ and chart moves of transition. By virtue of the conditions (i) and (ii) together with $n_{C}(\Gamma)=n_{C}\left(\Gamma^{\prime}\right), n_{L}(\Gamma)=n_{L}\left(\Gamma^{\prime}\right)$, $n_{0}^{+}\left(\Gamma \oplus N \Gamma_{0}\right)=n_{0}^{+}\left(\Gamma_{1}\right)$, and $n_{0}^{+}\left(\Gamma^{\prime} \oplus N \Gamma_{0}\right)=n_{0}^{+}\left(\Gamma_{1}^{\prime}\right)$, we conclude that $k=k^{\prime}$ because of $n_{0}^{+}\left(\Gamma_{0}\right) \neq 0$. Hence $\Gamma_{1}$ is transformed into $\Gamma_{1}^{\prime}$ by an ambient isotopy of $B$ relative to $b_{0}$, which means that $\Gamma \oplus N \Gamma_{0}$ is transformed into $\Gamma^{\prime} \oplus N \Gamma_{0}$ by chart moves of type $\mathrm{W}$, chart moves of transition, and ambient isotopies of $B$ relative to $b_{0}$. Therefore $f \# N f_{0}$ is (strictly) isomorphic to $f^{\prime} \# N f_{0}$ by Theorem 2.11.

We next prove the "only if" part. Take a non-negative integer $N$ so that $f$ \# $f_{0}$ is isomorphic to $f^{\prime} \# N f_{0}$. Since an isomorphism preserves numbers and types of vanishing cycles and signatures, we have

$$
n_{0}^{ \pm}\left(f \# N f_{0}\right)=n_{0}^{ \pm}\left(f^{\prime} \# N f_{0}\right), \quad n_{h}^{ \pm}\left(f \# N f_{0}\right)=n_{h}^{ \pm}\left(f^{\prime} \# N f_{0}\right)
$$

for every $h=1, \ldots,[g / 2]$, and

$$
\sigma\left(M \#_{F} N M_{0}\right)=\sigma\left(M^{\prime} \#_{F} N M_{0}\right) .
$$

The conditions (i), (ii), (iii) follow from additivity of $n_{0}^{ \pm}, n_{h}^{ \pm}, \sigma$ under fiber sum.

Definition 4.6 A Lefschetz fibration of genus $g$ over $S^{2}$ is called elementary if it contains exactly two singular fibers of type $\mathrm{I}^{+}$and of type $\mathrm{I}^{-}$which have the same vanishing cycles. A chart $L_{0}(i)$ in $S^{2}$ corresponds to an elementary Lefschetz fibration.

Remark 4.7 Two elementary Lefschetz fibrations of genus $g$ are isomorphic to each other. The total space of an elementary Lefschetz fibration of genus $g$ is diffeomorphic to $\Sigma_{g-1} \times S^{2} \# S^{1} \times S^{3}$.

We state the second of our main theorems. Let $B$ be a connected closed oriented surface and $f_{\star}: M_{\star} \rightarrow S^{2}$ an elementary Lefschetz fibration of genus $g$.

Theorem 4.8 Let $f: M \rightarrow B$ and $f^{\prime}: M^{\prime} \rightarrow B$ be Lefschetz fibrations of genus $g$. There exists a non-negative integer $N$ such that a fiber sum $f \# N f_{\star}$ is isomorphic to a fiber sum $f^{\prime} \# N f_{\star}$ if and only if the following conditions hold:

(i) $n_{0}^{ \pm}(f)=n_{0}^{ \pm}\left(f^{\prime}\right)$.

(ii) $n_{h}^{ \pm}(f)=n_{h}^{ \pm}\left(f^{\prime}\right)$ for every $h=1, \ldots,[g / 2]$.

(iii) $\sigma(M)=\sigma\left(M^{\prime}\right)$.

Remark 4.9 In contrast to Theorem 4.3, the isomorphism class of a fiber sum $f \#_{\Psi} f_{\star}$ of a Lefschetz fibration $f$ with an elementary Lefschetz fibration $f_{\star}$ depends on a choice of an orientation-preserving diffeomorphism $\Psi$ in general. 
Proof of Theorem 4.8 We only show the "if" part. The "only if" part is the same as that of the proof of Theorem 4.3.

Assume that $f$ and $f^{\prime}$ satisfy the conditions (i), (ii), and (iii). Let $\Gamma$ and $\Gamma^{\prime}$ be charts in $B$ corresponding to $f$ and $f^{\prime}$, respectively. It follows from the same argument as in the proof of Theorem 4.3 that $n_{C}(\Gamma)=n_{C}\left(\Gamma^{\prime}\right)$ and $n_{L}(\Gamma)=n_{L}\left(\Gamma^{\prime}\right)$. Let $N$ be an integer larger than both of the number of edges of $\Gamma$ and that of $\Gamma^{\prime}$. We construct the chart $\Gamma_{e}$ in $B$ for each $e \in E(\Gamma)$ as in the proof of Theorem 3.6. Taking the union of $\Gamma$ with $\Gamma_{e}$ for all $e \in E(\Gamma)$ and with $N-\# E(\Gamma)$ copies of $L_{1}(1)$, we obtain a new chart $\Gamma_{1}$ in $B$, which describes a fiber sum $f \# N f_{\star}$. Applying channel changes as in the proof of Theorem 3.6 and deaths of pairs of white vertices appropriately, we obtain a union $\Gamma_{2}$ of $n_{h}^{+}(\Gamma)$ copies of $L_{h}, n_{h}^{-}(\Gamma)$ copies of $L_{h}^{*},\left|n_{C}(\Gamma)\right|$ copies of $R_{C}$ (or $R_{C}^{*}$ ), $\left|n_{L}(\Gamma)\right|$ copies of $R_{L}$ (or $R_{L}^{*}$ ), and $k_{i}$ copies of $L_{0}(i)$ for some $k_{i}$, Similarly, $\Gamma^{\prime}$ is transformed into $\Gamma_{1}^{\prime}$, which describes a fiber sum $f^{\prime} \# N f_{\star}$, and then a union $\Gamma_{2}^{\prime}$ of $n_{h}^{+}\left(\Gamma^{\prime}\right)$ copies of $L_{h}, n_{h}^{-}\left(\Gamma^{\prime}\right)$ copies of $L_{h}^{*},\left|n_{C}\left(\Gamma^{\prime}\right)\right|$ copies of $R_{C}$ (or $R_{C}^{*}$ ), $\left|n_{L}\left(\Gamma^{\prime}\right)\right|$ copies of $R_{L}$ (or $R_{L}^{*}$ ), and $k_{i}^{\prime}$ copies of $L_{0}(i)$ for some $k_{i}^{\prime}$.

A similar argument on the number $n_{0}^{+}$as in the proof of Theorem 4.3 implies that $k_{0}+k_{1}+\cdots+k_{2 g}=k_{0}^{\prime}+k_{1}^{\prime}+\cdots+k_{2 g}^{\prime}$. Adding $\left|k_{i}-k_{i}^{\prime}\right|$ copies of $L_{0}(i)$ to either $\Gamma_{2}$ or $\Gamma_{2}^{\prime}$ if necessary, we may assume that $k_{i}=k_{i}^{\prime}$ for every $i \in\{0,1, \ldots, 2 g\}$. Hence $\Gamma_{2}$ is transformed into $\Gamma_{2}^{\prime}$ by an ambient isotopy of $B$ relative to $b_{0}$, which means that $f \# N f_{\star}$ is (strictly) isomorphic to $f^{\prime} \# N f_{\star}$ by Theorem 2.11.

Let $g$ be an integer greater than two and $B_{1}, \ldots, B_{r}$ connected closed oriented surfaces. We consider a Lefschetz fibration $f_{i}: M_{i} \rightarrow B_{i}$ of genus $g$ for each $i \in\{1, \ldots, r\}$, and a universal Lefschetz fibration $f_{0}: M_{0} \rightarrow S^{2}$ of genus $g$.

Proposition 4.10 For (possibly different) fiber sums $f$ and $f^{\prime}$ of $f_{1}, \ldots, f_{r}$, fiber sums $f \# f_{0}$ and $f^{\prime} \# f_{0}$ are isomorphic to each other.

Proof Let $\Gamma$ and $\Gamma^{\prime}$ be charts corresponding to $f$ and $f^{\prime}$. Since hoops surrounding a component of $\Gamma$ (and $\Gamma^{\prime}$ ) can be removed by use of the edges of $\Gamma_{0}$ as in Figure 19, $\Gamma \# \Gamma_{0}$ and $\Gamma^{\prime} \# \Gamma_{0}$ are transformed into the same chart.

Remark 4.11 Proposition 4.10 implies that there are many examples of non-isomorphic Lefschetz fibrations with the same base, the same fiber, and the same numbers of singular fibers of each type which become isomorphic after one stabilization. For example, the Lefschetz fibration on $E(n)_{K}$ constructed by Fintushel and Stern [9, Theorem 14] (see also Park and Yun [37]) for a fibered knot $K$ becomes isomorphic to that on $E(n)_{K^{\prime}}$ for another fibered knot $K^{\prime}$ of the same genus after one stabilization. 
Similar results hold for Lefschetz fibrations on $Y\left(n ; K_{1}, K_{2}\right)$ constructed by Fintushel and Stern [9, Section 7] (see also Park and Yun [38]) as well as fiber sums of (generalizations of) Matsumoto's fibration studied by Ozbagci and Stipsicz [36], Korkmaz [24; 25], and Okamori [35].

\section{Variations and problems}

In this section we discuss possible variations of chart description for Lefschetz fibrations. If we replace the triple $(\mathcal{X}, \mathcal{R}, \mathcal{S})$ defined in Section 2 with other triples, we obtain various chart descriptions for Lefschetz fibrations (see Kamada [20] and Hasegawa [15]).

We first choose large $\mathcal{X}, \mathcal{R}$, and $\mathcal{S}$. Let $\mathcal{X}$ be the set of right-handed Dehn twists along simple closed curves in $\Sigma_{g}$ and $\mathcal{S}$ the set of Dehn twists along non-trivial simple closed curves in $\Sigma_{g}$. By virtue of a theorem of Luo [29], $\langle\mathcal{X} \mid \mathcal{R}\rangle$ gives an infinite presentation of $\mathcal{M}_{g}$ for the set $\mathcal{R}$ of the following four kinds of words:

(0) Trivial relator $r_{T}:=a$, where $a$ is the Dehn twist along a trivial simple closed curve on $\Sigma_{g}$.

(1) Primitive braid relator $r_{P}:=b^{-1} a b c^{-1}$, where $a, b, c \in \mathcal{X}$ and the curve for $c$ is the image of the curve for $a$ by $b$.

(2) 2-chain relator $r_{C}:=\left(c_{2} c_{1}\right)^{6} d^{-1}$, where $c_{1}, c_{2}, d \in \mathcal{X}$ and the curves for $c_{1}$ and $c_{2}$ intersect transversely at one point and the curve for $d$ is the boundary curve of a regular neighborhood of the union of the curves for $c_{1}$ and $c_{2}$.

(3) Lantern relator $r_{L}:=\operatorname{cbad}_{4}^{-1} d_{3}^{-1} d_{2}^{-1} d_{1}^{-1}$, where $a, b, c, d_{1}, d_{2}, d_{3}, d_{4} \in \mathcal{X}$ and the curves for $a$ and $b$ intersect transversely at two points with algebraic intersection number zero, the curve for $c$ is obtained by resolving the intersections of these two curves, and the curves for $d_{1}, d_{2}, d_{3}, d_{4}$ are the boundary curves of a regular neighborhood of those for $a, b, c$.

Let $B$ be a connected closed oriented surface. Charts in $B$ for the triple $(\mathcal{X}, \mathcal{R}, \mathcal{S})$ defined above have white vertices of type $r_{T}^{ \pm 1}, r_{P}^{ \pm 1}, r_{C}^{ \pm 1}, r_{L}^{ \pm 1}$ (see Figure 26). For a chart $\Gamma$ in $B$, we denote the number of white vertices of type $r_{X}$ minus the number of white vertices of type $r_{X}^{-1}$ included in $\Gamma$ by $n_{X}(\Gamma)$, where $X=T, P, C, L$.

Proposition 5.1 The signature $\sigma(M)$ of the total space $M$ of a Lefschetz fibration $f: M \rightarrow B$ described by $\Gamma$ is equal to $-n_{T}(\Gamma)-7 n_{C}(\Gamma)+n_{L}(\Gamma)$.

Proof It is seen by a similar argument to the proof of Theorem 3.6. 

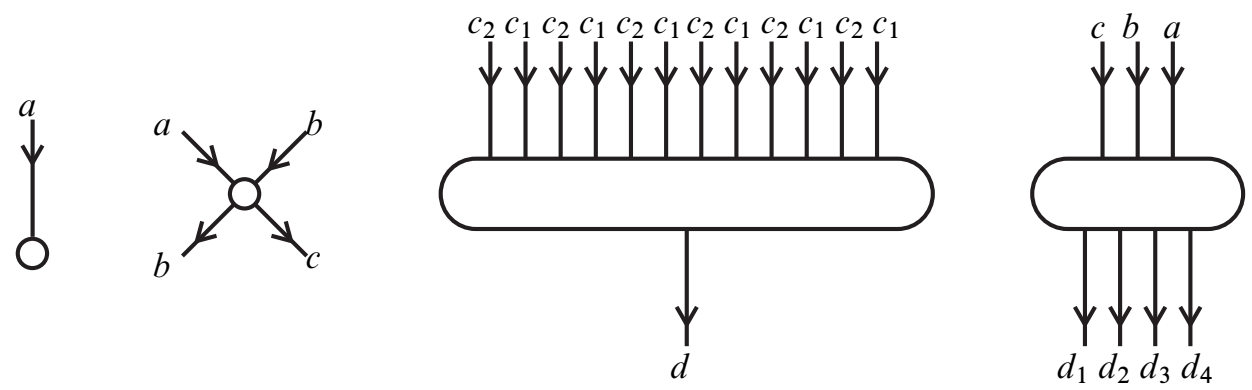

Figure 26: Vertices of type $r_{T}, r_{P}, r_{C}, r_{L}$

Example 5.2 Let $B$ be a connected closed oriented surface of genus 2 and $(\mathcal{X}, \mathcal{R}, \mathcal{S})$ the triple defined above for $g=3$. Let $a, b, c, d_{1}, d_{2}, d_{3}, d_{4}, c_{1}, c_{2}, c_{3}$ be right-handed Dehn twists along simple closed curves of the same names on $\Sigma_{3}$ depicted in Figure 27. We present $B$ as an octagon with opposite sides identified and consider a chart $\Gamma$ and loops $\gamma_{1}, \gamma_{2}, \gamma_{3}, \gamma_{4}, \gamma_{5}$ based at $b_{0}$ in $B$ as in Figure 28. We use a simplification of diagrams as in Figure 29(a) if the curves for $x, y \in \mathcal{X}$ intersect transversely at one point, and that as in Figure 29(b) if the curves for $x$ and $y$ are disjoint.

Since the intersection words of $\gamma_{1}, \gamma_{2}, \gamma_{3}, \gamma_{4}, \gamma_{5}$ with respect to $\Gamma$ are

$$
\begin{array}{lll}
w_{\Gamma}\left(\gamma_{1}\right)=d_{2}^{-1}, i & w_{\Gamma}\left(\gamma_{2}\right)=c_{3}^{-1} c^{-1} d_{2}^{-1} c_{3}^{-1}, \\
w_{\Gamma}\left(\gamma_{3}\right)=c_{1}^{-1} b^{-1} c_{2}^{-1} d_{3}^{-1} a^{-1} c_{2}^{-1} d_{4}^{-1} c_{1}^{-1}, & \\
w_{\Gamma}\left(\gamma_{4}\right)=d_{4} a^{-1}, & w_{\Gamma}\left(\gamma_{5}\right)=d_{1},
\end{array}
$$

a Lefschetz fibration $f: M \rightarrow B$ of genus 3 described by $\Gamma$ is isomorphic to the Lefschetz fibration constructed by Korkmaz and Ozbagci [26, Theorem 1.2]. $f$ has only one singular fiber and it is of type $\mathrm{I}^{+}$. We can compute the signature $\sigma(M)$ of the total space $M$ by Proposition 5.1 as

$$
\sigma(M)=n_{L}(\Gamma)=-1,
$$

which coincides with the value computed in [7, Proposition 14].
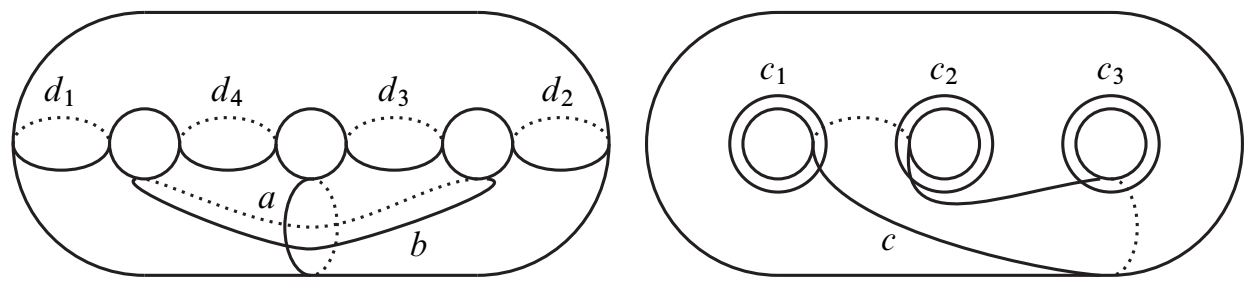

Figure 27: Simple closed curves on $\Sigma_{3}$ 


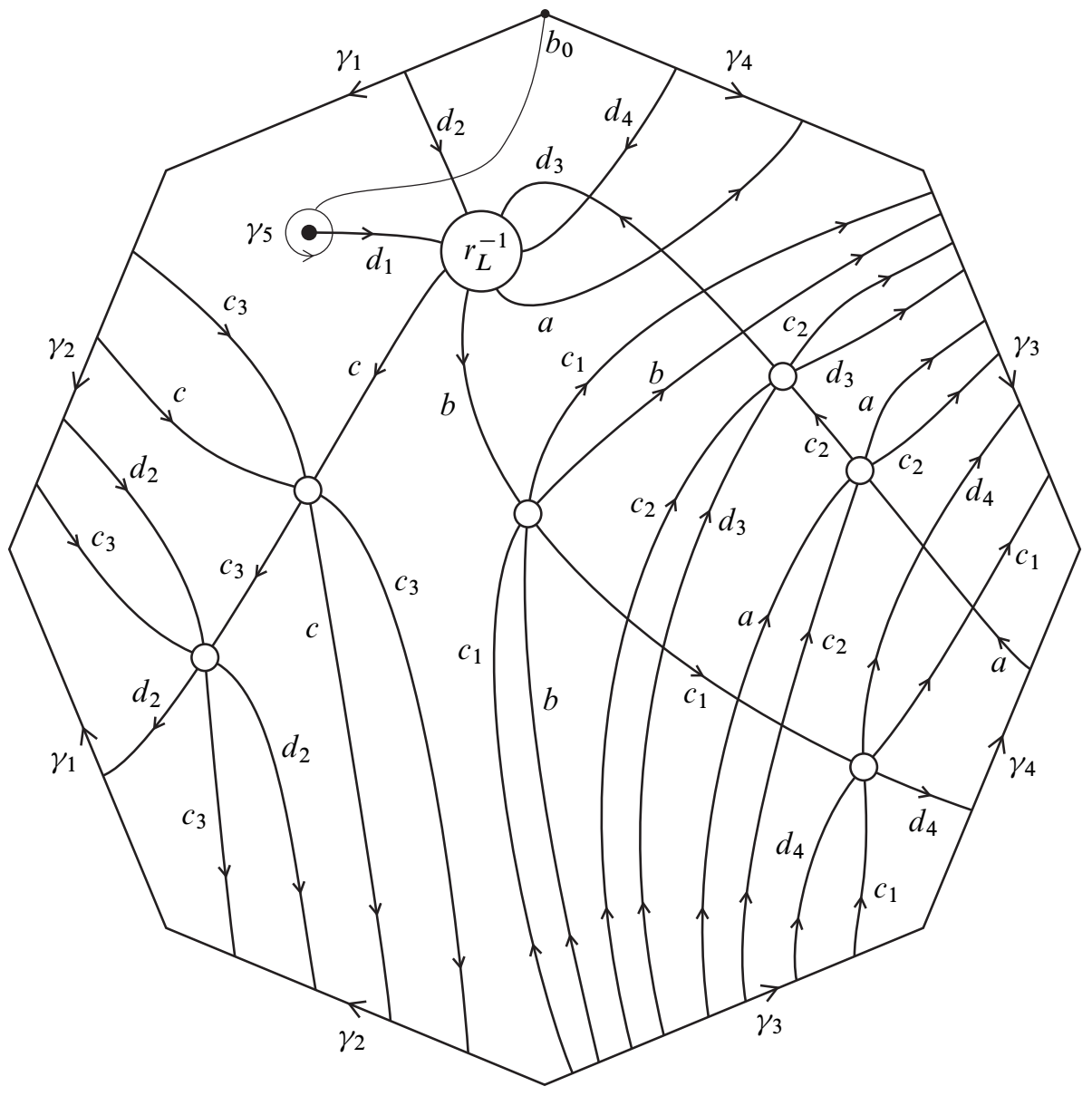

Figure 28: Chart for Lefschetz fibration of Korkmaz and Ozbagci

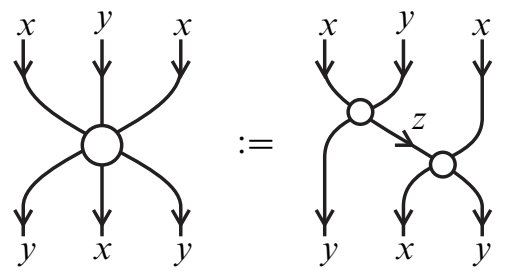

(a)

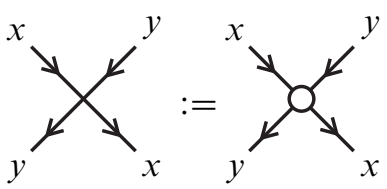

(b)

Figure 29: Simplification of vertices

Problem 5.3 Study various properties of Lefschetz fibrations by using chart descriptions of the triple $(\mathcal{X}, \mathcal{R}, \mathcal{S})$ defined above. 
We next mention chart descriptions for Lefschetz fibrations with bordered base and fiber. Kamada [20] gave a general theory for charts in a compact oriented surface with boundary. Various presentations of mapping class groups of surfaces with boundary have been investigated by researchers including Gervais [11], Labruère and Paris [28], Margalit and McCammond [30]. Combining these two kinds of studies, one can immediately obtain a chart description for Lefschetz fibrations with bordered base and fiber.

Problem 5.4 Make use of chart descriptions to study PALFs and Stein surfaces.

It would be worth considering compositions of monodromy representations with appropriate homomorphisms and charts corresponding to the compositions. For example, Hasegawa $[14 ; 15]$ adopted a homomorphism from the $m$-string braid group $B_{m}$ to the semi-direct product $\left(\mathbb{Z}_{2}\right)^{m} \times S_{m}$, while Endo and Kamada [6] used a standard epimorphism from the hyperelliptic mapping class group of a closed oriented surface of genus $g$ to the mapping class group of a sphere with $2 g+2$ marked points.

Problem 5.5 Consider chart descriptions for "nice" representations of mapping class groups to study invariants and classifications of Lefschetz fibrations.

Theorem 4.3 and Theorem 4.8 tell us that the numbers of singular fibers of all types and the signature of the total space completely determine the stable isomorphism class of a Lefschetz fibration with given base and fiber. Thus any numerical invariant of Lefschetz fibrations which is additive under fiber sum is determined by these invariants in principle.

Problem 5.6 Construct numerical invariants of Lefschetz fibrations which are not additive under fiber sum.

Nosaka [34] has recently defined an invariant which is not additive under fiber sum. Non-numerical invariants such as monodromy group would be also useful (see Matsumoto [32] and Park and Yun [37; 38]).

Acknowledgements The authors would like to thank the referees for their helpful suggestions and corrections. The first author was partially supported by JSPS KAKENHI grants number 21540079,25400082 . The third author was partially supported by JSPS KAKENHI grants number 21340015, 26287013. The fourth author was partially supported by JSPS KAKENHI grants number 21740042, 26400082. 


\section{References}

[1] D Auroux, Fiber sums of genus 2 Lefschetz fibrations, Turkish J. Math. 27 (2003) 1-10 MR1975329

[2] D Auroux, A stable classification of Lefschetz fibrations, Geom. Topol. 9 (2005) 203217 MR2115673

[3] J Barge, É Ghys, Cocycles d'Euler et de Maslov, Math. Ann. 294 (1992) 235-265 MR1183404

[4] R İ Baykur, S Kamada, Classification of broken Lefschetz fibrations with small fiber genera, J. Math. Soc. Japan 67 (2015) 877-901 MR3376572

[5] H Endo, S Kamada, Chart description for hyperelliptic Lefschetz fibrations and their stabilization, preprint (2013) arXiv:1306.2707 to appear in Topology Appl.

[6] H Endo, S Kamada, Counting Dirac braids and hyperelliptic Lefschetz fibrations, preprint (2015) arXiv:1508.07687

[7] H Endo, M Korkmaz, D Kotschick, B Ozbagci, A Stipsicz, Commutators, Lefschetz fibrations and the signatures of surface bundles, Topology 41 (2002) 961-977 MR1923994

[8] H Endo, S Nagami, Signature of relations in mapping class groups and nonholomorphic Lefschetz fibrations, Trans. Amer. Math. Soc. 357 (2005) 3179-3199 MR2135741

[9] R Fintushel, R J Stern, Families of simply connected 4-manifolds with the same Seiberg-Witten invariants, Topology 43 (2004) 1449-1467 MR2081432

[10] J-M Gambaudo, É Ghys, Braids and signatures, Bull. Soc. Math. France 133 (2005) 541-579 MR2233695

[11] S Gervais, A finite presentation of the mapping class group of a punctured surface, Topology 40 (2001) 703-725 MR1851559

[12] P M Gilmer, G Masbaum, Maslov index, lagrangians, mapping class groups and TQFT, Forum Math. 25 (2013) 1067-1106 MR3100961

[13] R E Gompf, A I Stipsicz, 4-manifolds and Kirby calculus, Graduate Studies in Mathematics 20, Amer. Math. Soc. (1999) MR1707327

[14] I Hasegawa, A certain linear representation of the classical braid group and its application to surface braids, Math. Proc. Cambridge Philos. Soc. 141 (2006) 287-301 MR2265876

[15] I Hasegawa, Chart descriptions of monodromy representations on oriented closed surfaces, $\mathrm{PhD}$ thesis, Univ. Tokyo (2006)

[16] K Hayano, On genus-1 simplified broken Lefschetz fibrations, Algebr. Geom. Topol. 11 (2011) 1267-1322 MR2801419 
[17] S Kamada, Surfaces in $\mathbf{R}^{4}$ of braid index three are ribbon, J. Knot Theory Ramifications 1 (1992) 137-160 MR1164113

[18] S Kamada, An observation of surface braids via chart description, J. Knot Theory Ramifications 5 (1996) 517-529 MR1406718

[19] S Kamada, Braid and knot theory in dimension four, Mathematical Surveys and Monographs 95, Amer. Math. Soc. (2002) MR1900979

[20] S Kamada, Graphic descriptions of monodromy representations, Topology Appl. 154 (2007) 1430-1446 MR2310476

[21] S Kamada, Chart description for genus-two Lefschetz fibrations and a theorem on their stabilization, Topology Appl. 159 (2012) 1041-1051 MR2876710

[22] S Kamada, Y Matsumoto, T Matumoto, K Waki, Chart description and a new proof of the classification theorem of genus one Lefschetz fibrations, J. Math. Soc. Japan 57 (2005) 537-555 MR2123244

[23] A Kas, On the handlebody decomposition associated to a Lefschetz fibration, Pacific J. Math. 89 (1980) 89-104 MR596919

[24] M Korkmaz, Noncomplex smooth 4-manifolds with Lefschetz fibrations, Internat. Math. Res. Notices (2001) 115-128 MR1810689

[25] M Korkmaz, Lefschetz fibrations and an invariant of finitely presented groups, Int. Math. Res. Not. 2009 (2009) 1547-1572 MR2500970

[26] M Korkmaz, B Ozbagci, Minimal number of singular fibers in a Lefschetz fibration, Proc. Amer. Math. Soc. 129 (2001) 1545-1549 MR1713513

[27] Y Kuno, Meyer functions and the signatures of fibered 4-manifolds, preprint (2012) arXiv:1204.1701

[28] C Labruère, L Paris, Presentations for the punctured mapping class groups in terms of Artin groups, Algebr. Geom. Topol. 1 (2001) 73-114 MR1805936

[29] F Luo, A presentation of the mapping class groups, Math. Res. Lett. 4 (1997) 735-739 MR1484704

[30] D Margalit, J McCammond, Geometric presentations for the pure braid group, J. Knot Theory Ramifications 18 (2009) 1-20 MR2490001

[31] Y Matsumoto, Diffeomorphism types of elliptic surfaces, Topology 25 (1986) 549-563 MR862439

[32] Y Matsumoto, Lefschetz fibrations of genus two-A topological approach, from: “Topology and Teichmüller spaces”, (S Kojima, Y Matsumoto, K Saito, M Seppälä, editors), World Scientific, River Edge, NJ (1996) 123-148 MR1659687

[33] W Meyer, Die Signatur von Flächenbündeln, Math. Ann. 201 (1973) 239-264 MR0331382 
[34] T Nosaka, Bilinear-form invariants of Lefschetz fibrations over the 2-sphere, preprint (2014)

[35] K Okamori, A genus-2 Lefschetz fibration on an exotic $\mathbb{C P}^{2} \# 9 \overline{\mathbb{C P}}^{2}$, master's thesis, Osaka University (2011)

[36] B Ozbagci, A I Stipsicz, Noncomplex smooth 4-manifolds with genus-2 Lefschetz fibrations, Proc. Amer. Math. Soc. 128 (2000) 3125-3128 MR1670411

[37] J Park, K-H Yun, Nonisomorphic Lefschetz fibrations on knot surgery 4-manifolds, Math. Ann. 345 (2009) 581-597 MR2534109

[38] J Park, K-H Yun, Lefschetz fibration structures on knot surgery 4-manifolds, Michigan Math. J. 60 (2011) 525-544 MR2861087

[39] V G Turaev, The first symplectic Chern class and Maslov indices, Zap. Nauchn. Sem. Leningrad. Otdel. Mat. Inst. Steklov. 143 (1985) 110-129 MR806563 In Russian; translated in J. Soviet Math 37 (1987) 1115-1127

[40] B Wajnryb, A simple presentation for the mapping class group of an orientable surface, Israel J. Math. 45 (1983) 157-174 MR719117

[41] B Wajnryb, An elementary approach to the mapping class group of a surface, Geom. Topol. 3 (1999) 405-466 MR1726532

[42] C T C Wall, Non-additivity of the signature, Invent. Math. 7 (1969) 269-274 MR0246311

Department of Mathematics, Tokyo Institute of Technology

2-12-1 Oh-okayama, Meguro-ku, Tokyo 152-8551, Japan

Ministry of Health, Labour and Welfare

1-2-2 Kasumigaseki, Chiyoda-ku, Tokyo 100-8916, Japan

Department of Mathematics, Osaka City University

3-3-138 Sugimoto, Sumiyoshi-ku, Osaka 558-8585, Japan

Department of Mathematics, Tokyo Gakugei University

4-1-1 Nukuikita-machi, Koganei-shi, Tokyo 184-8501, Japan

endo@math.titech.ac.jp, i.ch.braid.lf@gmail.com,

skamada@sci.osaka-cu.ac.jp, kotanaka@u-gakugei.ac.jp

Received: 17 April 2015 
